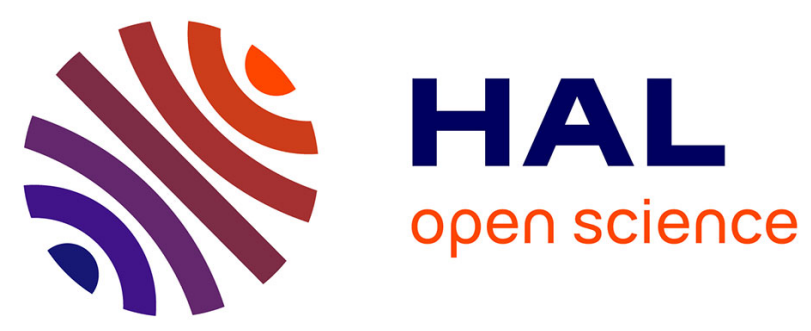

\title{
Chitosan-based nanoparticles for in vivo delivery of interfering agents including siRNA
}

\author{
Christine Vauthier, Christelle Zandanel, Anne Laure Ramon
}

\section{To cite this version:}

Christine Vauthier, Christelle Zandanel, Anne Laure Ramon. Chitosan-based nanoparticles for in vivo delivery of interfering agents including siRNA. Current Opinion in Colloid \& Interface Science, 2013, 18 (5), pp.406 - 418. 10.1016/j.cocis.2013.06.005 . hal-03194518

\section{HAL Id: hal-03194518 \\ https://hal.science/hal-03194518}

Submitted on 9 Apr 2021

HAL is a multi-disciplinary open access archive for the deposit and dissemination of scientific research documents, whether they are published or not. The documents may come from teaching and research institutions in France or abroad, or from public or private research centers.
L'archive ouverte pluridisciplinaire HAL, est destinée au dépôt et à la diffusion de documents scientifiques de niveau recherche, publiés ou non, émanant des établissements d'enseignement et de recherche français ou étrangers, des laboratoires publics ou privés. 


\title{
Chitosan-based nanoparticles for in vivo delivery of interfering agents including siRNA.
}

\section{Christine VAUTHIER ${ }^{\mathrm{a}, \mathrm{b} *}$, Christelle ZANDANEL ${ }^{\mathrm{a}, \mathrm{b}}$, Anne Laure RAMON RA,c $^{\mathrm{a}, \mathrm{c}}$}

\author{
${ }^{a}$ Univ Paris-Sud, Faculté de Pharmacie, 5, rue J.B. Clément, Châtenay-Malabry, France \\ ${ }^{\mathrm{b}}$ CNRS UMR 8612, Institut Galien Paris-Sud, 5, rue J.B.Clément, Châtenay-Malabry, France \\ c CNRS UMR 8203 Vectorologie et thérapeutiques anticancéreuses, 114 rue Edouard \\ Vaillant, Villejuif, France
}

Published in: Curr Opin Colloid Interface Sci. 2013; 18(5): 406-418. DOI: 10.1016/j.cocis.2013.06.005

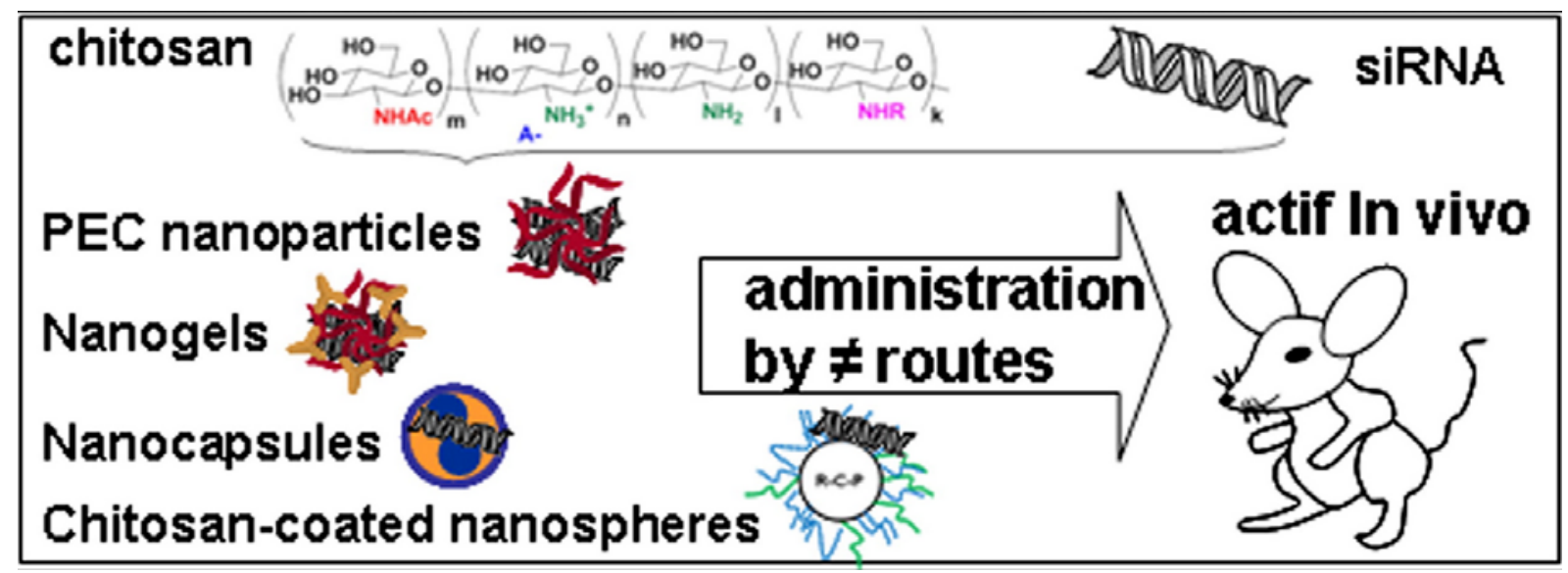

*Corresponding author

Christine Vauthier, Faculté de Pharmacie, Institut Galien Paris-Sud, University of Paris Sud, 5 Rue J.B. Clément, F 92296 Châtenay-Malabry France 5, Rue J.B. Clément, F-92296 Chatenay-Malabry Cedex, France.

E-mail: Christine.vauthier@u-psud.fr 


\begin{abstract}
The potential of siRNA to knock down expression of genes has been identified as an exiting strategy for specific treatments of disease associated genes. However, their clinical development is pended to the achievement of their effective intracellular delivery in the target cells in vivo. So far, this was a bottleneck for fast development of siRNA in clinics because of their high enzymatic susceptibility in biological media and their poor intracellular uptake. The realization of therapeutic potential of the RNA interference approach strongly depended on the rational design of safe and effective carriers. This review considers carriers made of chitosan-based nanoparticles. It reports the methods of synthesis and the interactions of siRNA with chitosan which is at the basis of the association, stability and delivery to cells of siRNA with these carriers. Results of evaluations of the interference activity produced in vitro and in vivo by the interfering molecule delivered with chitosan-based nanoparticle carriers are discussed. As pointed out from different examples, the remarkable efficacy of the chitosanbased nanoparticles to deliver active interfering agents in vivo and to achieve a successful systemic delivery including by oral administration are very encouraging. Although we are still in the early stage of developments, it can be expected that results reported so far paved the road to stimulate further developments and strengthen their clinical application perspectives.
\end{abstract}

Keywords: polyelectrolyte complex, nanogel, nanocapsules, nanoparticles, chitosan, siRNA, interference, gene silencing. 


\section{Introduction}

siRNA interference remains a recent discovery. While the RNA interference phenomenon was first described 23 years ago, the elucidation of the mechanism occurred at the drawn of the 21 st century [1]. The power of small interfering RNA (siRNA) to knock down expression of genes was immediately identified as an exiting new potential approach for the development of very specific treatments of disease-associated genes. This covers a wide range of pathologies. Therapeutic strategies based on an interference effect have the potential to control viral infections, cancer development and degenerative diseases [2,3]. The development of clinically relevant siRNA to achieve a reliable and specific gene silencing activity has progressed extremely rapidly. Several efficient siRNA have reached advanced stages of clinical developments when local treatments can be applied [2]. SiRNA are RNA molecules that are active by themselves directly after being transfected into cells. Their clinical development is pending to the achievement of their effective intracellular delivery in target cells in in vivo conditions. Their chemical nature is a bottleneck for the developments of treatments especially those based on a systemic delivery. The high enzymatic degradation susceptibility of siRNA in biological media and their polyanionic nature which contribute to their poor cellular uptake constitute major technical hurdle that prevent so far their appearance in the arsenal of molecules for a wide range of antiviral and antitumoral therapies. The realization of the therapeutic potential of RNA interference strongly depends on the rational design of safe and efficient delivery systems making possible the delivery of the siRNA to target cells in vivo after a systemic administration. Several potential delivery systems consist of viral particles, but it is generally admitted that effective non-viral delivery system for siRNA will be preferred for obvious safety reasons [2,4,5]. Several types of synthetic non-viral materials have been found suitable to achieve effective delivery of siRNA into cells in vitro [5-8]. Unfortunately, they cannot be further developed to achieve in vivo delivery of siRNA due to their toxicological profile despite many efforts made to improve their safety profiles $[7,8]$. Nevertheless, a few of them including lipofectamine and polyethyleneimine were developed as transfection agents for in vitro use. With time, they became references for the evaluation of the transfection given by new compounds in in vitro experiments. Today, chitosan, which is a polysaccharide, is the component which seems to be unanimously adopted for the development of nanoparticle delivery systems for nucleic acids. It is used in more then $80 \%$ of nanoparticles designed for genes and interfering components [9-11]. These systems are assumed to present a good compromise between safety and transfection efficiency. They have 
extensively been used for gene delivery $[12,13]$. Based on this experience they were tested as delivery systems for siRNA. The intense activity justifies that there are already several reviews on the subject especially covering the delivery of DNA by chitosan-based delivery systems [13]. A few review papers report research carried on the application of an interference strategy thanks to the delivery of siRNA with chitosan based delivery systems [14-16]. The aim of the present review was to describe the nanoparticles designed with chitosan that were suggested as delivery systems for siRNA and to give an overview on the results obtained so far on the in vitro and in vivo evaluations of the interference effects. For this, the review was balanced between the physico-chemical aspects of chitosan-based nanoparticles as colloids loaded with siRNA and those of the use of biological models to evaluate the potential of the approach delivering the siRNA with different delivery systems. The methods for the preparation of the nanoparticles, the characteristics and performance in term of siRNA loading are presented in the first part of this review. As the association of the siRNA with chitosan-based nanoparticles mainly results from interactions with chitosan, a second part of this review discusses the different parameters that governs this interaction including on the thermodynamic point of view. The last part of the review summarises interference activities reported using the nanoparticles as it was evaluated considering different levels in the delivery challenge. This will review in vitro data in which the potentials of the delivery systems are evaluated in conditions allowing direct contact with cells. For the in vivo evaluations, the results were presented considering the different routes of administrations that were tested in the view to produce a local or a systemic effect.

\section{Obtaining chitosan-based nanoparticles for siRNA delivery}

Different types of nanoparticles were designed with chitosan to achieve in vivo delivery of interfering agents including siRNA. Methods considering the association or entrapment of siRNA in the nanoparticles are exploiting the polyanionic character of these molecules. The negative charges come from the phosphate group included in each nucleotide composing the two strands of the interfering agent. This polyanionic character is exploited to form polyelectrolyte complexes with polycations. Although the first polycation designed in this purpose was poly(ethylenimine) (PEI) [6] which still inspires several works [7], today, chitosan and its derivatives have become the major component used to design delivery systems for interfering molecules. As explained in the reviews of Yuan et al. [11] and Raftery 
et al. [13], the main advantages of chitosan are its superior safety in term of biocompatibility and degradation which is combined with a satisfactory transfection efficacy considering the delivery of nucleic acids. The arguments were based on the numbers of good indicators coming from the FDA which recognized chitosan as a generally recognize as safe for applications in the food industry and in the formulation of wound dressing materials. It was also based on the encouraging results provided from tests evaluating their toxicity under the form of nanoparticles in numerous studies having considered their applications for nucleic acid delivery. However, it is noteworthy the evaluation of the biocompatibility of chitosan under the form of nanoparticles remains to be evaluated in a suitable framework to evaluate their safety prior to the start of clinical testing [17]. The structure of chitosan and principal characteristics influencing its interactions with siRNA are summarized in figure 1. As it was proposed for the delivery of genes, modified chitosans were also used to formulate nanoparticle systems for the delivery of siRNA. The range of modifications suggested so far is narrower compared with those proposed for gene delivery (Figure 1) [18].

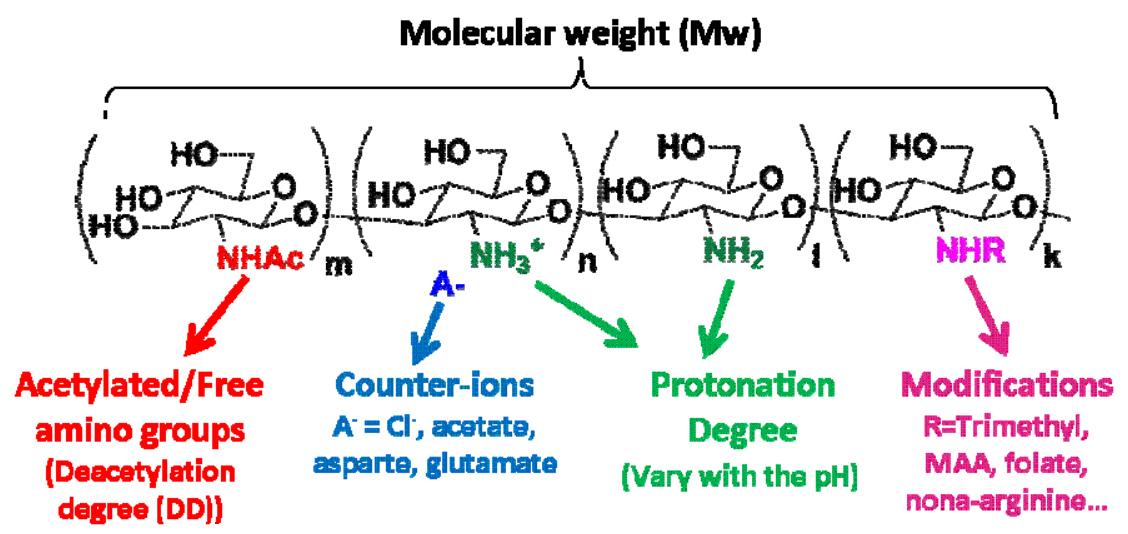

Figure 1: Structure of chitosan and parameters influencing interactions with siRNA

In general, modified chitosan are used to modulate the functionality of the carrier to adjust delivery properties to those required for the desired application [12]. The different types of nanoparticles made of chitosan and chitosan derivatives designed to achieve in vivo delivery of siRNA can be classified as polyelectrolyte complexes (PEC) nanoparticles, nanogels, nanocapsules and core-corona nanospheres. It can be noted that PEC nanoparticles and nanogels were the subject of intense research for the delivery of DNA [13] and of siRNA [14] This part of the review will present principles for their formation, their structures and their principal characteristics in term of size, zeta potential and encapsulation efficiency. 


\subsection{Formation of nanosized polyelectrolyte complexes between SiRNA and chitosan}

Polyelectrolyte complexes (PEC) form spontaneously by mixing polymers of opposite charges. PEC of nanometer size range can occur under certain conditions defined by the ratios of molecular weight between the two polyelectrolytes and their respective concentration [19]. Due to their polyanionic character, nucleic acids can form PEC with chitosan at acidic $\mathrm{pH}$ where chitosan is protonated (Figure 2) [20].

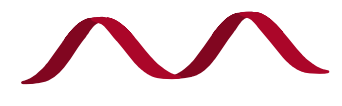

chitosan
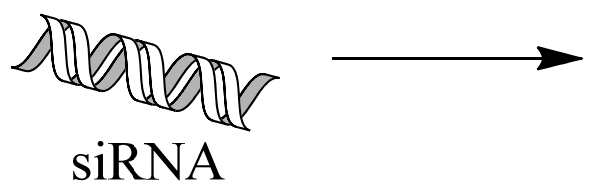

Figure 2: Polyelectrolyte complexation process

This method of preparation of nanosized PEC complex was mainly applied for the encapsulation of siRNA with high encapsulation efficiency (EE) (above 95\%) [21]. In general, the nanoparticles recovered are positively charged indicating that the siRNA is entrapped inside the PEC. The average diameters varying between 200 and $500 \mathrm{~nm}$ increased with the concentration of chitosan used to form the PEC nanoparticles [20]. The molecular weight of chitosan influences the average diameter of the particles especially at high concentrations (200-300 $\mu \mathrm{g} / \mathrm{mL}$ of chitosan) [20] but the influence is less marked on the zeta potential of the particles. At high concentration of chitosan also, there was no influence of the type of chitosan, hydrochloride or glutamate, on the average diameter and zeta potential while significant differences appeared at concentrations below $100 \mathrm{mg} / \mathrm{mL}$ [20].

Trimethylchitosan (TMC) is a derivative of chitosan used to form PEC nanoparticles with siRNA. One of the major advantages is the obtaining of PEC complex with siRNA over a wide range of $\mathrm{pH}$ compared with the parent non modified chitosan $[18,23,24]$. Indeed, while chitosan which is partially protonated at neutral $\mathrm{pH}(\mathrm{pKa} 6.3-6.4)$, the protonation of the amino group is $\mathrm{pH}$ independent when it is trimethylated which favor the solubility of the macromolecule event at physiological $\mathrm{pH}$ and the formation of complex with siRNA. The size of PEC nanoparticles obtained with trimethylchitosan is reduced compared with those 
formed with the non modified chitosan. This is favorable for an easier diffusion of the carrier in tissues in vivo compared with larger particles. Because the protonation of trimethylchitosan is independent to the $\mathrm{pH}$ of the dispersing medium, another advantage is the positive zeta potential shown by the PEC nanoparticles (around $+20 \mathrm{mV}$ ) even at neutral or slightly basic $\mathrm{pH}$. This contributes to the good colloidal stability of the dispersions.

Trimethylchitosan can also be used to form $\mathrm{pH}$ responsive PEC nanoparticles thanks to the further addition of segments of oligo(methacrylic acid) (MAA) [23]. After formation of PEC nanoparticles by complexation with siRNA, the release of the siRNA can be triggered by a drop of $\mathrm{pH}$ of the surrounding media. The release of the entrapped siRNA can occur in very well controlled place in the organism where the nanoparticles reached the conditions of $\mathrm{pH}$ for the release. The average diameter of the PEC nanoparticles formed by the association of siRNA and oligo(methacrylic acid) grafted trimethylchitosan is greatly influenced by the content in oligo(methacrylic acid) in the copolymer. It can range between 200 and more than $1000 \mathrm{~nm}$ [23]. The stability of the dispersion is also influenced by the content in MAA copolymer and chitosan.

\subsection{Nanogels}

Nanogels can be defined as reticulated PEC nanoparticles. The only difference between PEC nanoparticles and nanogels is the ionic reticulation of the complex formed between the interfering agent and chitosan with oligo-anions or polyanions. The most used reticulants are TriPhenylPhosphine (TPP) and Hyaluronic Acid (HA) respectively [25] (Figure 3). The nucleic acid is associated with nanogels according to two methods. The preferred method consists in mixing chitosan, siRNA and the reticulant (TPP/HA) together. This one-pot synthesis leads to the highest encapsulation efficiencies (>95\%) (Figure 3a). A second method of loading of nanogels consists in an adsorption of the siRNA on already formed particles resulting from the complexation of chitosan with the oligo or polyanion as illustrated in figure $3 \mathrm{~b}$. In this case, the encapsulation efficiency is lower compared with the loading method based on the entrapment of the siRNA and depends greatly on the nature of the counter ion of chitosan (83-90\% for chitosan glutamate, 59-72\% for chitosan hydrochloride) [18]. Nanogels formed by ionic gelation can be obtained with chitosan, N-modified chitosan (TMC, thiolated TMC [250]; mannose-modified trimethyl chitosan-cysteine [26], O-modified chitosan (insertion of PEG in C6 position) [27], RGD-labeled chitosan [28]. 


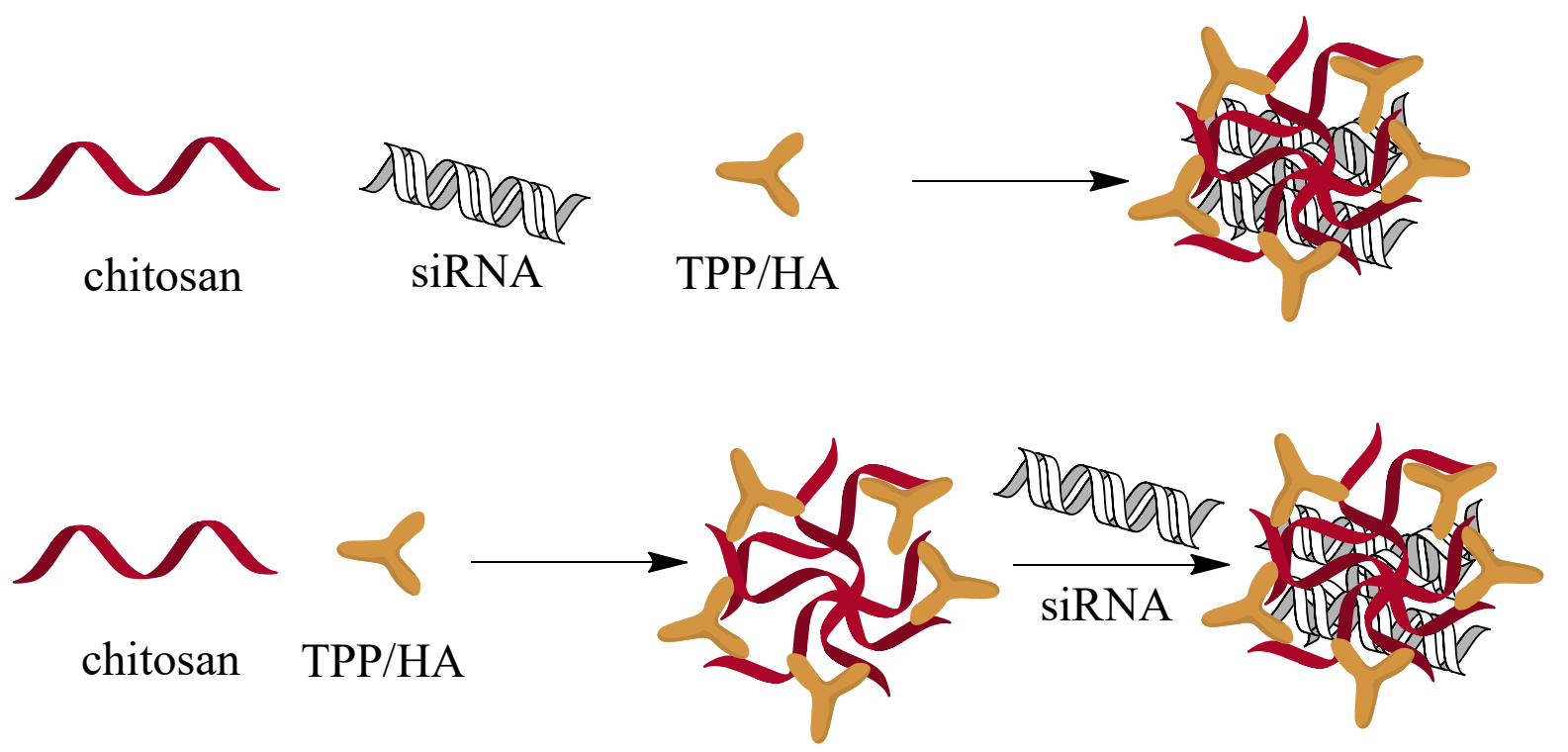

Figure 3: Preparation of nanogels by ionic gelation. A: Incorporation of the siRNA during the formation of nanogel, $B$ : association of the siRNA with nanogels formed from the reticulation of chitosan with triphenylphosphine (TPP) or hyaluronic acid (HA)

It was shown that the reticulation has no influence on the average diameter (100 to $230 \mathrm{~nm}$ ) of the nanoparticles and their zeta potential $(+6$ to $+27 \mathrm{mV})[20]$. They were comparable to those of corresponding PEC nanoparticles (average diameter ranging from 100 to $250 \mathrm{~nm}$, zeta potential from +5 to $+24 \mathrm{mV}$ ).

\subsection{Nanocapsules}

Nanocapsules are nanosized containers consisting in a tiny droplet of liquid surrounded by a polymer envelope. Two types of nanocapsules can be distinguished depending on the nature of the liquid entrapped in the container which can be either an aqueous solution or oil. Water containing nanocapsules designed with a poly(D,L-lactide-co-glycolide) (PLGA) envelope allowed to encapsulate siRNA within the inside of the container [29]. These nanocapsules were produced by the emulsion solvent evaporation process carried out from a double emulsion (Figure 4). In this relatively gentle method, a water-in-oil (w/o) emulsion is prepared first with PLGA dissolved in the organic phase and aqueous solution of poly(VinylAlcool) (PVA) containing the siRNA. This emulsion is then emulsified in a new aqueous phase containing PVA and eventually chitosan (Figure 4). During the evaporation of the organic solvent, the envelope of the nanocapsules formed by precipitation of the PLGA at the interface found between the two aqueous phases of the double emulsion. 


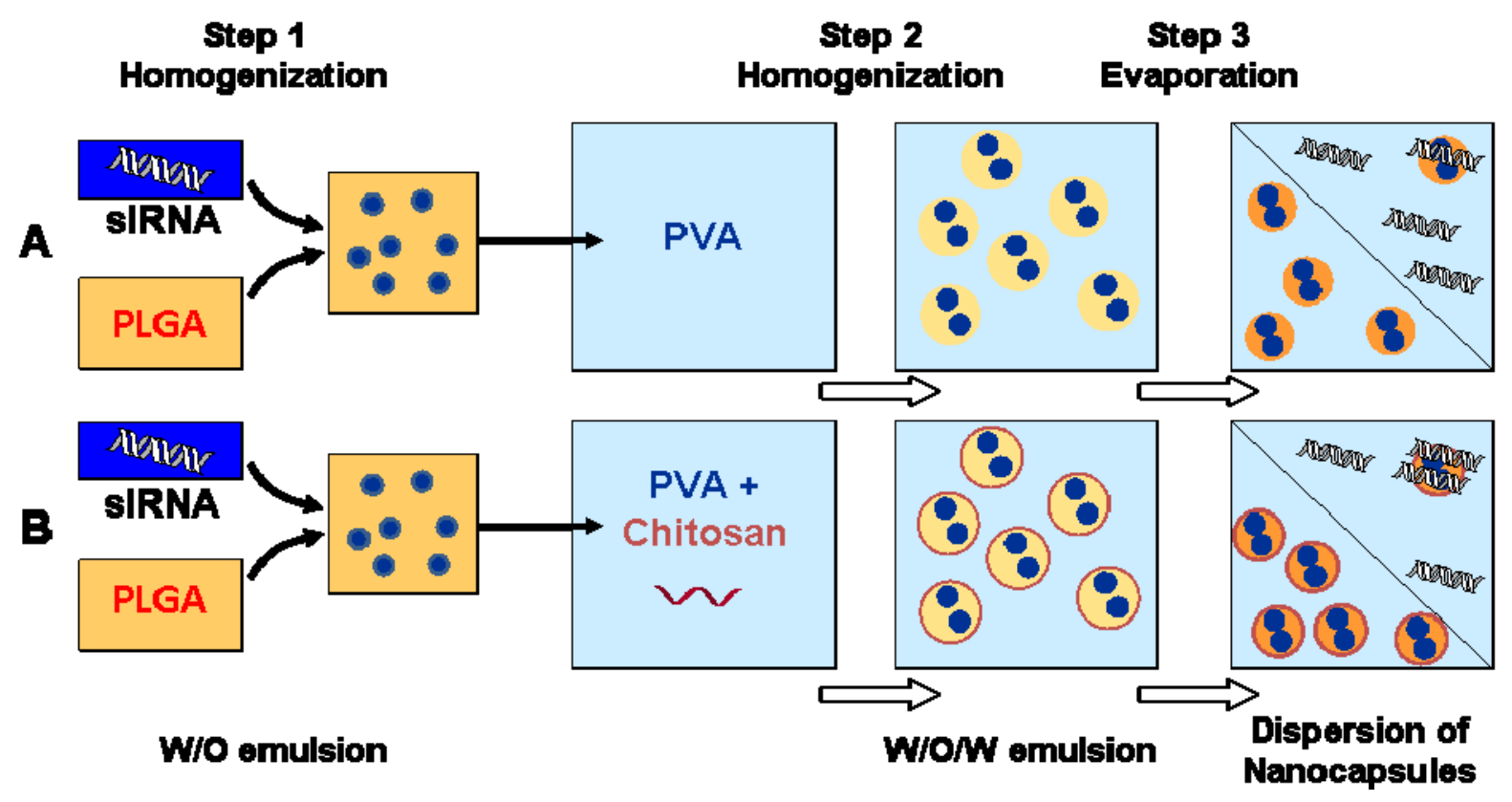

Figure 4: Principle of the formation of aqueous containing nanocapsules without (A) or with (B) chitosan at the surface. Aqueous phases are indicated in blue while organic phase is shown in orange. The upper part of the frame showing the dispersion of nanocapsules illustrates the performance of the nanocapsules in term of association of siRNA while the lower part gives a scheme of the expected structure of the nanocapsules.

The average diameter of the nanocapsules was highly influenced by the concentration of PLGA and PVA, by the composition in lactate and glycolide of PLGA, the organic/aqueous phase volume ratio and the homogenization process used to prepare the emulsion (stirring time, homogenization time and sonication time) [30]. SiRNA added in the inner aqueous phase were poorly encapsulated by this method with encapsulation efficiency of only $28 \%$ [31]. The encapsulation efficiency was significantly improvement (up to $44 \%$ ) by adding chitosan in the outer aqueous phase of the double emulsion. As indicated by the zeta potential of the nanocapsules which was positive, chitosan adsorbed on the nanocapsules surface. The average diameter of the chitosan-coated nanocapsules $(352 \mathrm{~nm})$ was slightly increased compared to the diameter showed by the non-coated nanocapsules $(317 \mathrm{~nm})$ [31]. The layer of chitosan formed at the surface of the nanocapsules improved the encapsulation of the siRNA and also protected siRNA against degradation by nucleases. This layer of chitosan was also useful to reduce the burst release of the siRNA which was observed with the non-modified PLGA nanocapsules [31]. By dissolving a dye in the organic phase during preparation of the emulsion, the nanocapsules could be labeled without significant modification of the average diameter and zeta potential. 
Water-containing nanocapsules were obtained by interfacial polymerization of IsoButylCyanoAcrylate (PIBCA) in water-in-oil microemulsion. siRNA designed to target the junction of the EWS/Fli1 junction oncongene was encapsulated with a high encapsulation efficiency (>95\%) [32]. The average diameter of the nanocapsules was around $320 \mathrm{~nm}$ and the zeta potential was negative $(-28 \mathrm{mV})$.

Finally, siRNAs were associated with oil-containing nanocapsules. The nanocapsules were obtained by chemical reticulation of N-((2-hydroxy-3-trimethylammonium)propyl) chitosan chloride with glutaraldehyde. The N-((2-hydroxy-3-trimethylammonium)propyl) chitosan chloride was dissolved in the aqueous phase of an oil-in-water-in-oil (o/w/o) emulsion. The siRNA was associated with the nanocapsules envelope after formation of the nanocapsules. Subsequent addition of N-((2-hydroxy-3-trimethylammonium)propyl) chitosan chloride was required to prevent premature release of the siRNA and degradation by nucleases. The oily core of the nanocapsules was used to co-encapsulate paclitaxel, a major anticancer agent used in clinics in treatments of cancer by chemotherapy [33]. In general, the siRNA was loaded on the preformed nanocapsules at a siRNA/nanocapsules ratio of $1 / 80$. The average diameter of the nanocapsules was $130 \mathrm{~nm}$.

\subsection{Polymeric nanospheres}

Polymeric nanospheres can be defined as solid colloidal particles. In general, those designed for interfering agent delivery display a core-corona structure. SiRNA carriers developed on this model were composed of a Poly(AlkylCyanoAcrylate) (PACA) core surrounded by a corona of chitosan which served as host for the siRNA. The nanospheres can be synthesized either by anionic emulsion polymerization or by redox radical polymerization. In both cases, the initiation of the polymerization of the alkylcyanoacrylate monomer is initiated on the polysaccharide but the mechanism of initiation of the polymerization differs (Figure 5). As a consequence, the copolymers between PACA and chitosan which formed displayed different structures influencing the conformation of the chitosan chains in the nanosphere corona [3438]. Although the chemical composition of these nanospheres do not differ significantly, the difference in the spacial arrangement of the chitosan chains on the nanosphere surface influences their interactions with blood proteins that can influence their respective in vivo fate. The average diameter of the nanospheres recovered was influenced by the mechanism of the polymerization (radical vs anionic). Smaller nanospheres were generated by AEP compared with RREP while both syntheses were performed using the same chitosan $[35,38]$. 


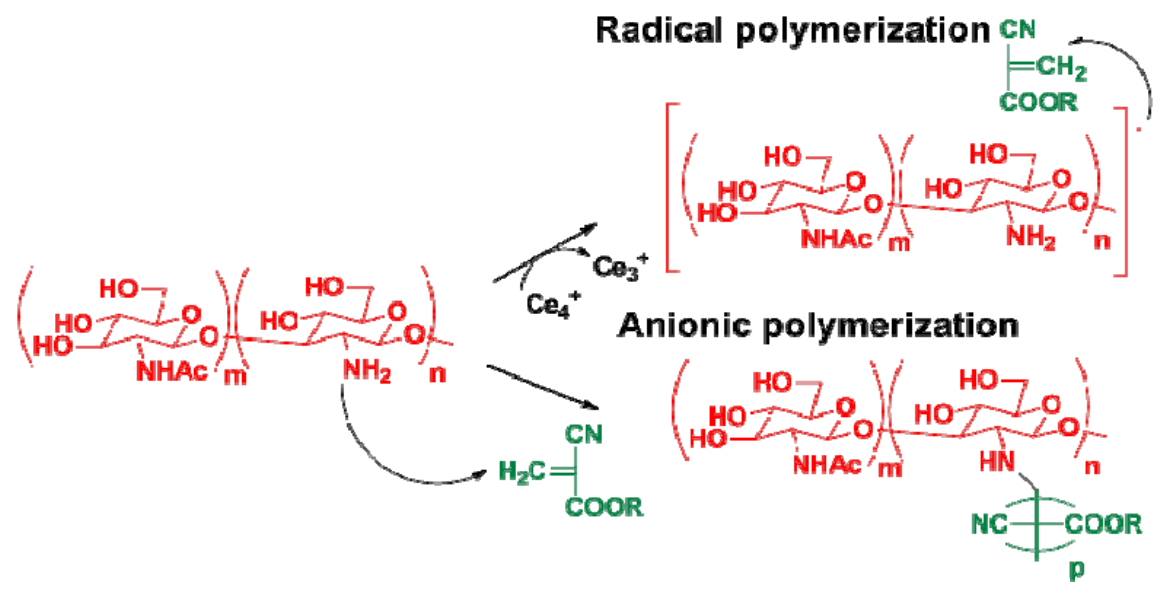

Figure 5: Mechanisms of polymerization of chitosan with Poly(IsoButylCyanoAcrylate) (adapted from [38])

The average diameter also increased with the molecular weight of non modified or thiolated chitosan [36] and depended on the type of alkylcyanoacrylate monomer used. The diameter of Poly(IsoHexylCyanoAcrylate) (PIHCA) nanospheres was larger than that of Poly(IsoButylCyanoAcrylate) (PIBCA) nanospheres using a chitosan having a molecular weight of $20 \mathrm{KDa}$. The size of the nanospheres was reduced without loosing the stability of the dispersion by adding pluronic in the polymerization medium. The addition of pluronic combined with an increase of the temperature of the polymerization allowed the obtaining of nanospheres as small as $50 \mathrm{~nm}$ including a corona composed of both pluronic and chitosan (Figure 6,7) [37-39].
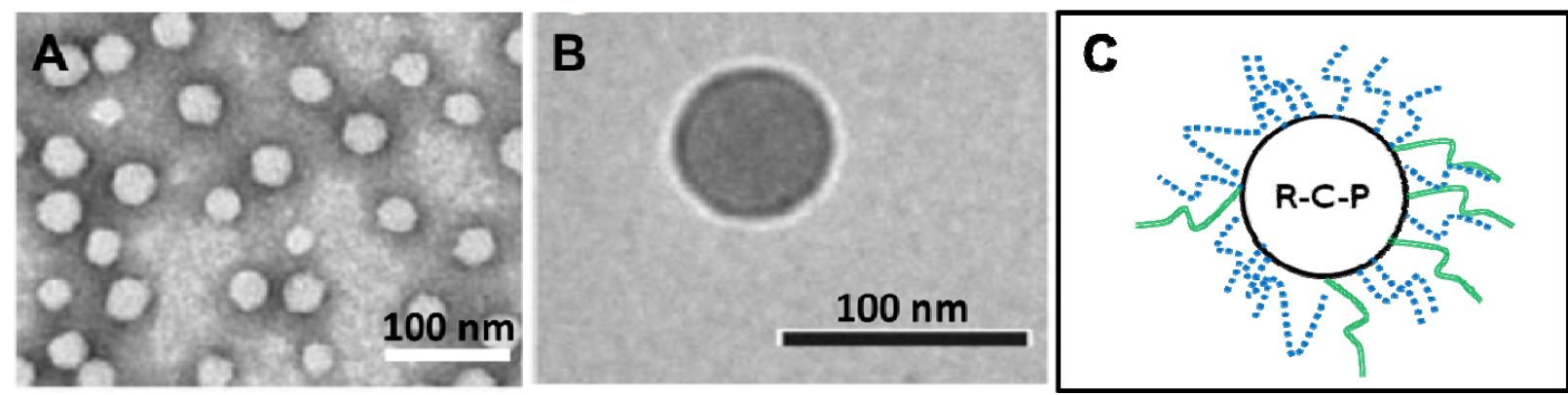

Figure 6: Transmission electron microscopy of chitosan coated PIBCA nanospheres prepared in presence of pluronic F68 $3 \%$ A and B: transmission electron micrograph at different magnifications. $C$ : Scheme showing the arrangement of the chitosan (in green) and pluronic (in blue) chains in the corona of the chitosan coated PIBCA nanospheres. 


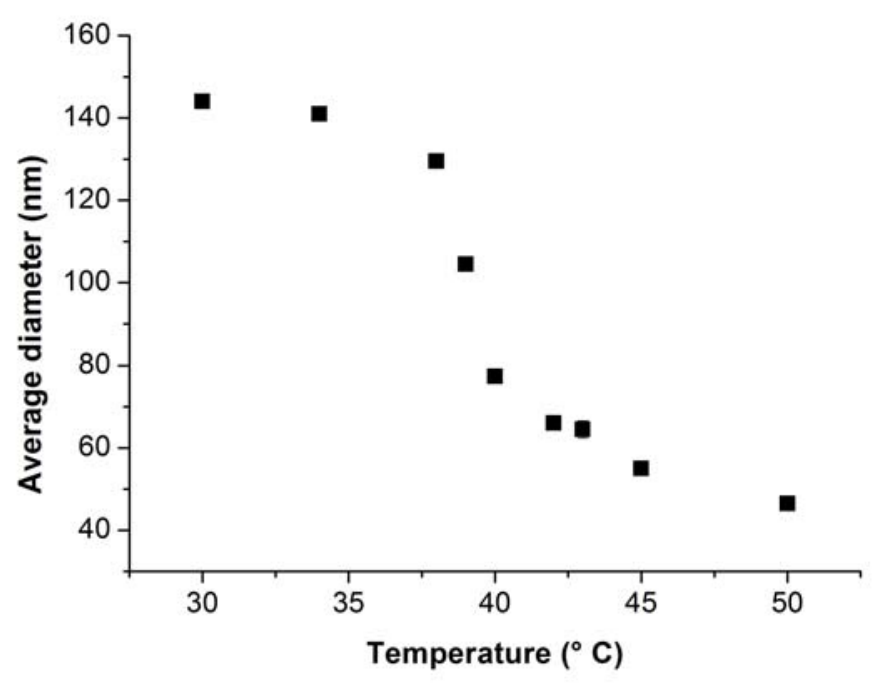

Figure 7: Evolution of the average diameter of the nanoparticles with the temperature of the polymerization medium. Preparation was performed with $1.3 \%$ of chitosan and $3 \%$ of Pluronic F-68.

This small size was desired to achieve in vivo delivery of siRNA to tumors from an intravenous injection thanks to a passive targeting of tumoral tissue by the Enhanced Permeability and Retention (EPR) effect. It is noteworthy that this method of preparation of the nanospheres was scaled up from lab scale preparation $(10 \mathrm{~mL})$ to batch of $500 \mathrm{~mL}$ without loosing the characteristics of the nanospheres. SiRNAs were loaded on the nanospheres by adsorption on the chitosan corona. This was achieved without modification of the average diameter of the nanospheres. The optimal loading (encapsulation efficiency $>95 \%$ ) was achieved for a nanosphere/siRNA ratio of 50. This mode of association was efficient to provide a good protection of the siRNA against degradation by the nucleases including in in vivo conditions [37]. Another interesting property of these nanospheres is that they can be labelled with a fluorescent probe. A fluorescent monomer is copolymerized with the alkylcyanoacrylate monomer providing with a stable labelling of the nanosphere core. This method was suitable to preserve the properties of the nanosphere corona as acknowledge by identical thermodynamic characteristics of the interactions between the siRNA and the labelled and non-labelled nanospheres as well as their similar profile of interactions with the complement system (Figure 8) [40]. 


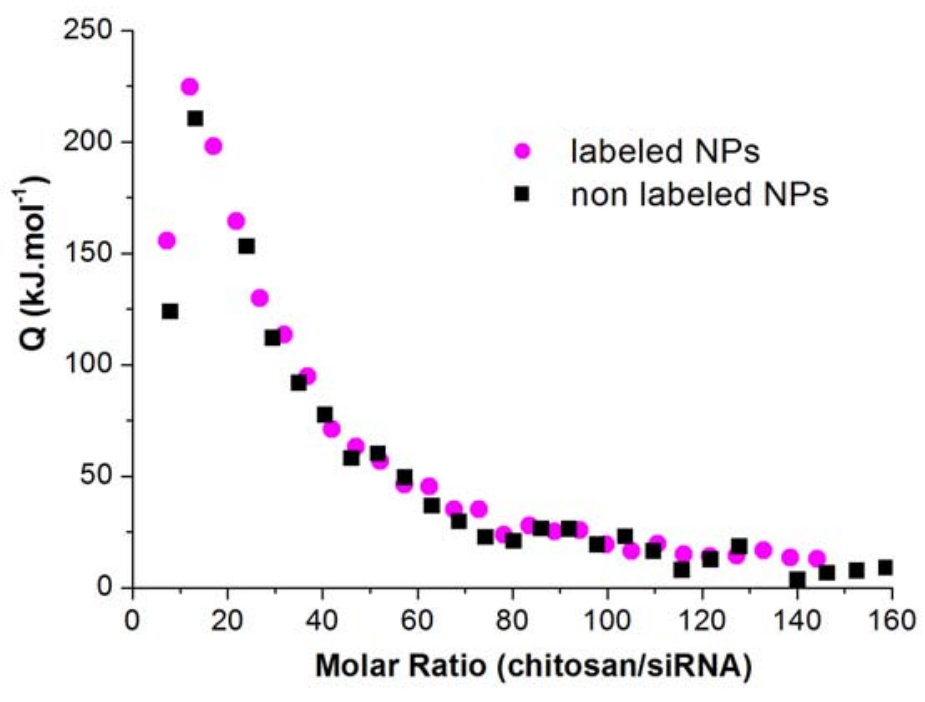

Figure 8: Profiles of the energy exchanges recorded during isothermal titration calorimetry (ITC) of non-labelled (black squares) and labelled nanoparticles (pink circles) with siRNA. The similarity of the profiles indicated that the two types of nanoparticles were identical regarding their capacity to interact with the siRNA.

\section{Characteristics of the interactions between chitosan and SiRNA.}

Interactions of siRNA with chitosan govern many properties of the drug delivery system $[14,15]$. First, they control the association of the siRNA with the chitosan-based nanoparticles. Second, they control the stability of the complex which in turn controls the protection of siRNA against enzymatic degradation since the siRNA remain protected when it forms a complex with the nanoparticles. Finally, these interactions are also important to control the release of the siRNA.

Despite the fact that chitosan seems to play a major role in the loading of chitosan-based nanoparticles with interfering agents including siRNA and in the stability of the complex formed, only few studies have investigated the thermodynamic characteristics and strength of interactions taking place between siRNA and chitosan. Binding enthalpies and binding constants were evaluated by isothermal titration calorimetry (ITC) considering the association of siRNA with PEC nanoparticles and chitosan coated PIBCA nanospheres [41]. From these studies, it appeared that the characteristics of the interactions between siRNA and chitosan did not vary with modification of the molecular weight of chitosan in the range 44 to $143 \mathrm{KDa}$ during formation of PEC nanoparticles at $\mathrm{pH} 5$ [41]. Considering the association of siRNA with the chitosan coated PIBCA nanospheres, it was shown that it resulted from a 
spontaneous phenomenon [42]. The enthalpy of the interaction depended on the nature of the counter ion associated with the chitosan molecule. The enthalpy of interaction also varied with the sequence of the siRNA (Figure 9). Comparing two siRNA with known sequences, the enthalpy increased while the AU/CG ratio characterizing the composition in bases of the siRNA decreased. The composition of the siRNA may have an influence on the hydrophobic interactions that are also involved in the association of siRNA with chitosan [42].

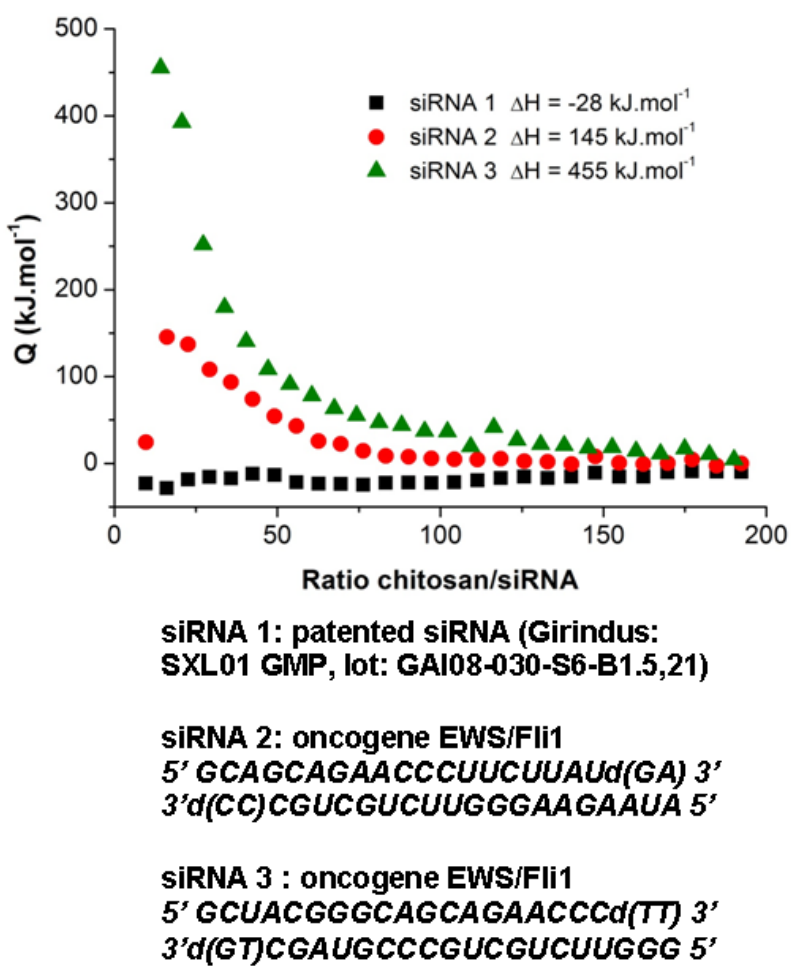

Figure 9: Influence of the siRNA sequence on the binding enthalpy measured association with chitosan coated PIBCA nanospheres. The nanospheres were prepared with chitosan hydrochloride $20 \mathrm{KDa}$ and dispersed in MilliQ ${ }^{\circledR}$ water.

The strength of the interaction between siRNA and chitosan chains stranded at the surface of gold nanospheres was evaluated by atomic force microscopy (AFM) [43]. These measures were performed at different $\mathrm{pH}$ considering a chitosan of $13 \mathrm{KDa}$ and a degree of deacetylation of $77 \%$. The strongest interactions occurred at $\mathrm{pH} 4.1$ showing a multimodal distribution of adhesive forces compared with interactions at $\mathrm{pH} 7.4$ characterized by a low force frequency. This was attributed to the occurrence of a larger amount of positive charges on the chitosan chain while the $\mathrm{pH}$ was decreased. Only the few studies reported above have provided with a quantitative analysis of the influence of several parameters on the association of siRNA with chitosan which mediate their association with the drug carrier. Other parameters influencing association of siRNA with chitosan-based nanoparticle systems were 
identified from qualitative observations. They were mainly drawn from experiments designed to evaluate the encapsulation efficiency and from studies evaluating the stability of the siRNA-nanoparticle assembly. Collectively, it appears from the different works that all parameters influencing the number of positive charges brought in the system by chitosan have an influence on the association with the siRNA. These includes the concentration in chitosan [20], the degree of protonation of chitosan which is linked with the degree of deacetylation and with the $\mathrm{pH}$ of the medium [21,44], the degree of deacetylation [45] and the modification of the amino groups of chitosan by using different types of substituent $[23,24,46]$. Although most works leading to this conclusion were achieved from the obtaining of siRNA loaded PEC nanoparticles [20,45], similar observations were reported considering the preparation of siRNA loaded nanogels [21] and other types of nanoparticles where siRNA associated with the particles thanks to a direct interaction with chitosan. All these parameters also influence the stability of the association of the siRNA with the chitosan-based nanoparticles hence the release of the siRNA from the nanoparticles and in turn the protection of the siRNA against degradation by nucleases.

Besides, the number of positive charges, the N/P ratio which corresponds to the ratio between positive and negative functions involved in the interactions also greatly influences the association of siRNA with chitosan-based nanoparticles based on the interactions with chitosan as well as the stability of this association. Finally, stability of the PEC nanoparticles is enhanced with chitosan of high molecular weight $(\mathrm{Mw}>12 \mathrm{KDa})$ [45,47]. A scheme summarizing the sense of variation of the different parameters on the influence on the association of siRNA with PEC nanoparticles and of the stability of the association is proposed in figure 10 .

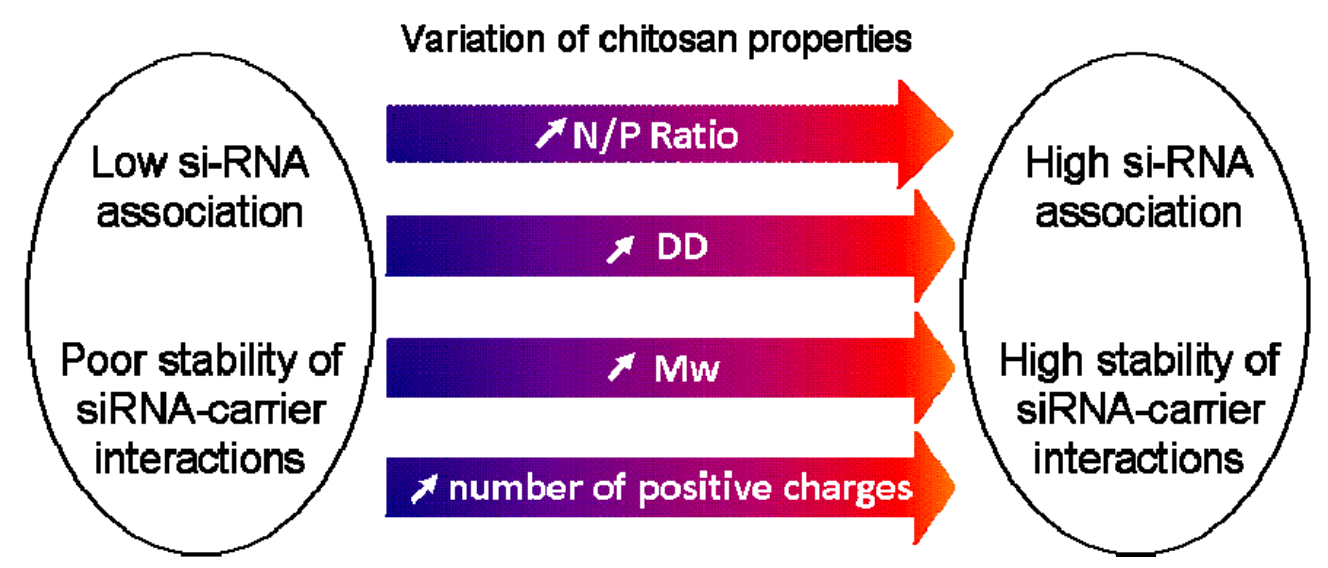

Figure 10: Impact of $M w, D$, number of positive charges and N/P ratio on the association of siRNA with chitosan-based nanoparticles and on the stability of the association. 
The difficulty found with formulations based on the association of siRNA thanks to the formation of a complex with chitosan is to find the right compromise between the parameters that promote the formation of the siRNA-chitosan complex, those which ensure the stability required for the delivery of the siRNA to the target cell and to those that achieve the dissociation of the complex inside the cells. In general, parameters requested to promote the association of the siRNA and the stability of the complex are in opposition with those needed for the delivery of the siRNA (Figure 10). A balance needs to be found. In this perspective, the use of modified chitosan offers a wide range of possibilities to modulate properties of siRNA-chitosan complex including to design of $\mathrm{pH}$ sensitive complexes that will release the siRNA while the $\mathrm{pH}$ of the environment dropped down [23]. The influence of each of these parameters was analyzed in details in the review proposed by Al-Qadi et al. [14].

Considering the obtaining of siRNA-loaded water-containing PLGA nanocapsules, the siRNA is assumed to be entrapped within the heart of the nano-container. In this case, chitosan is not required for the formation of the nanocapsules which actually form even in the absence of chitosan. However, a minimal concentration of chitosan $0.0067 \% \mathrm{w} / \mathrm{v}$ was required to increase the amount of siRNA that actually associated with the nanocapsules and to prevent premature release of the siRNA from the nanocapsules [48,49]. Regarding the association of siRNA with chitosan-coated nanospheres, this is generally achieved by adsorption of the siRNA on already prepared nanospheres $[30,33,36]$. It could be calculated that the chitosan coated PIBCA nanospheres can associate an average number of 120 siRNA per particle [37]. In general, the adsorption of siRNA is promoted at acidic $\mathrm{pH}$ while at $\mathrm{pH} 7.4$ the electrostatic forces involved in the association are weaker because of a lower ionization of the chitosan chain [30]. A major difference can be pointed out between the chitosan coated PLGA nanospheres and the PACA nanospheres. Chitosan was simply adsorbed on the surface of the PLGA nanospheres while it was covalently attached on the surface of the PIBCA nanospheres. This can make a huge difference in terms of stability of the association of the siRNA with the nanospheres especially once the carrier is administered in vivo.

\section{In vitro evaluation of siRNA delivery to cells}

A few studies have reported the use of chitosan-based delivery systems to explore their potential to achieve transfection of siRNA into cells considering in vitro experiments. In these 
experiments, the carrier loaded with the siRNA is incubated with cells expressing the target mRNA which means that there is almost no barrier between the delivery system and the cell membrane. Then, it is expected that these conditions are the most favourable to observe the interference activity of a given siRNA if all other conditions are met to silent the expression of the target mRNA. Precisely, to obtain an interfering effect, the siRNA needs to reach the target mRNA which is located in the cell cytoplasm. At first sight, the general positive charge observed with chitosan-based nanoparticles designed for siRNA delivery is a favourable property to promote interactions with cell membranes which are generally negatively charged thanks to the presence of negatively charged proteoglycans. Thus, it should promote the cellular uptake of the carrier thanks to these primary interactions. However, the zeta potential of the carrier is determined while the nanoparticles are dispersed in media free of proteins. The charge may greatly differ in media containing proteins which reflect more accurately the conditions found in culture media supplemented with serum and in vivo. Proteins that generally adsorbed on the surface of nanoparticles can mask the initial positive charged hence reduces potential interactions of the carrier with the proteoglycans of the cell membranes. A contribution of the electrostatic charge for the success of the delivery method may exist. However, it is suggested that other pathways are also contributing to the internalization of siRNA within the cells. As shown considering chitosan-based delivery systems for gene therapy, they are believed to involve receptors [13,50,51]. Additionally, receptor mediated endocytosis can be promoted by grafting a ligand at the surface of the carrier. For instance, the grafting of mannose residues on trimethyl-chitosan-cysteine nanoparticles promoted the internalization of siRNA in macrophages through a pathway that was independent from the clathrin route [52]. Once in the cells, the siRNA must be released from the carrier and reached the cytoplasm without being degraded. A mechanism based on a proton sponge effect that allowed intact DNA to escape from endosomes after delivery with chitosan-based nanoparticles to cells was described in the case of gene delivery. It is assumed that a similar mechanism could apply with siRNA. Obviously more studies on mechanisms of siRNA delivery to cells using chitosan-based nanoparticles are needed. This will be needed to clarify the role of the different parameters of the formulations that are influencing success of producing an interfering activity. Indeed, at present, all parameters already identified for the formation and stability of the chitosan-based nanoparticles for the delivery of siRNA were found to influence success in transfection experiments [16]. This will also be needed to understand discrepancies that were pointed out in some studies that mentioned unsuccessful transfection in vitro while the siRNA delivered in vivo with the nanoparticles was able to 
generate a clear interference effect [37,38]. Despite the need of deeper investigations to have a better view on the delivery mechanisms of siRNA with chitosan-based nanoparticles, evaluation of the interfering activity produced by these systems have been started using a few types of nanoparticles which were tested on different models. As it will be discussed below, chitosan/siRNA-based delivery systems were evaluated to silence expression of proteins such as the cell membrane efflux pump P-glycoprotein (P-gp) responsible for drug resistances, and proteins responsible for tumour neo-vascularisation including the Vascular Endothelial Growth Factor (VEGF) and tumour growth like Akt-1.

Malmo et al. [53] used chitosan/siRNA PEC nanoparticles to silence the expression of the Pgp in the blood brain barrier. The rational behind this strategy was to improve the passage of drugs from the bloodstream to the brain. To explore this hypothesis, the authors have carried on transfection experiments of the siRNA directed against the P-gp on a model of rat brain endothelial cell line, the RBE4, which is commonly used as a BBB model. The chitosan/siRNA PEC nanoparticles were efficient to silence the P-gp but the level of efficacy depended on the N/P ratio used to form the PEC nanoparticles. The amount of internalized siRNA delivered with the PEC nanoparticles decreased by increasing the N/P ratio but high $\mathrm{N} / \mathrm{P}$ ratios were requested to produce the highest knock down of P-gp expression. In vitro, the reduction of P-gp expression was acknowledged by a successful transfection of the siRNA into the RBE4 cells and by a reduction of the efflux capacity of the cells. It was also shown that doxorubicin, which was used as a model drug in this study, could be better delivered to the cells leading to an increase of its biological activity. As concluded by the authors, results from this work suggested that transfection of a siRNA designed to silence P-gp to the endothelial cells of the blood brain barrier could be used as a general strategy to improve the delivery of drugs to the brain which remains a bottleneck to efficient treatment for many types of diseases [53]. Nevertheless, in vivo studies will be necessary to fully demonstrate the usefulness of siRNA/chitosan PEC nanoparticles to block the expression of the P-gp and increase the efficiency of the delivery of actives in the brain. The validation of the delivery method will also need to explore the application of this strategy to deliver drugs to the brain considering a series of relevant drugs in addition to doxorubicin.

Fernandes et al. [47] have investigated the in vitro transfection efficiency of a siRNA directed against Sjogren Syndrome antigen (SSB, GenBank accession number NM 009278) using chitosan/siRNA PEC nanoparticles on different cell lines, the HeLa cell line from cervical carcinoma, the OV-3 cell line an ovarian carcinoma cell line and an osteosarcoma cell line 
MG-63. Two of the cell lines, the HeLa and OV-3 cell lines, expressed folate receptors on the cell membrane in contrast with the third cell line, MG-63. So the PEC nanoparticles were prepared with chitosan bearing folic acid residues to investigate the efficiency of a targeting approach. It was assumed that the addition of a targeting ligand on the siRNA delivery system may potentiate the transfection efficacy of the siRNA in the corresponding targeted cells. The transfection efficiency was actually increased in the two types of folate receptor positive cell lines, HeLa and OV-3 cells, using folate chitosan/siRNA PEC nanoparticles. These nanoparticles which were decorated with folate groups on their surface, were designed to specifically target cancer cells that express a folate receptor on their cell membrane [54]. In contrast, the presence of the targeting ligand on the PEC nanoparticles showed no effect on the transfection efficiency of the siRNA in folate receptor negative cell line, MG-63 cells [47]. The cytotoxicity of the chitosan used to prepare chitosan/siRNA PEC nanoparticles was studied on the HeLa cells with regards to the molecular weight. The low molecular weight chitosans, 2 and $5 \mathrm{kDa}$, have a higher cytotoxicity (IC50 $=0.21 \mathrm{mg} / \mathrm{mL}$ ) compared to 10,25 and $50 \mathrm{kDa}$ chitosan. With an IC50 of $2.2 \mathrm{mg} / \mathrm{mL}$, chitosans 10 and $25 \mathrm{kDa}$ were the less cytotoxic [47]. Using chitosan with a molecular weight above $10 \mathrm{kDa}$, the viability of the cells was independent on the chitosan/siRNA ratio composing the PEC nanoparticles up to a ratio of 100. Interestingly, the higher transfection efficiency observed with the folate-chitosan PEC nanoparticles was also accompanied with the lower cytotoxicity of the targeted siRNA delivery system to folate receptor positive cells. This should encourage further development of strategies considering cell specific delivery of siRNA by targeting the folate receptor overexpressed in various diseased cells which include several types of cancer cells and cells of inflammatory diseases such as arthritis.

Beside the use of chitosan/siRNA PEC nanoparticles, nanogels composed of chitosan, siRNA and TPP were evaluated for their capacity to silence a reporting gene, pGL3-luciferase, in CHO K1 and HEK 293 cells [20]. An interesting aim of this work was to investigate the influence of the method of association of the siRNA with the nanogel as well as the influence of the type of chitosan. Results of the in vitro studies performed on both types of cells showed that the method of preparation of the nanogel hence the method of association of the siRNA with the nanogel played an important role on the silencing effect. The addition of siRNA together with TPP to the chitosan solution during the ionic gelation process (Figure 3A) showed better gene silencing activities compared with the method of association of the siRNA based on the adsorption of the siRNA on preformed nanogels (Figure 3B) on both CHO-K1 
and HEK-293 cells lines. Additional results showed that nanogels showed a higher potential to achieve efficient transfection and delivery of the siRNA into cells than the corresponding chitosan/siRNA PEC nanoparticles. It is suggested that the higher performance highlighted using the nanogels was due to the high binding capacity and loading efficiency of the nanogels in siRNA. The reticulation and the entrapment in the core of the nanogel improved siRNA-nanogel association and stability of the siRNA against degradation which could be other favourable factors contributing to the higher transfection efficacy reported with nanogels compared with PEC nanoparticles. Interestingly, nanogels designed with chitosan having different counterions, glutamate or hydrochloride, also showed different interference activities. The nanogels prepared with glutamate chitosan showed the highest post transfection gene silencing effect. Besides the fact that nanogels appeared superior to achieve an interference activity by delivering siRNA to cells, they also appeared less cytotoxic compared with the other transfection systems tested in the same conditions which included chitosan/siRNA PEC nanoparticles and lipofectamine, the reference agent used in transfection experiments. To achieve specific delivery to CD4+ T cells, nanogels specifically targeting $\mathrm{T}$ cells were designed using a CD7-specific single-chain antibody (scFvCD7) [55]. The antibody was coupled with chitosan by carbodiimide chemistry prior to the preparation of the siRNA loaded nanogel crosslinked with polyguluronate. While no dramatic modification of the formation of the siRNA-loaded nanogels was reported, the presence of the targeting ligand increased the uptake of the nanogels by the target cells. This was accompanied by a reduction of the expression of the CD4 receptor on the cell surface and by a reduction of the amount of the corresponding mRNA. All the results converged with the conclusion that the delivery of siRNA with targeted nanogels improved cell specificity of the delivery and the efficacy of the gene silencing strategy. These results together with those discussed above considering folate receptor targeted PEC nanoparticles [47] are very encouraging. They were obtained from experiments performed in vitro on cell cultures. They should now be translated in vivo.

\section{In vivo evaluation of siRNA delivery with chitosan-based nanoparticles}

Several examples of in vivo delivery of siRNA using chitosan-based delivery systems can be found in the literature. Interestingly, these systems were tested to deliver interfering agents by different routes of administration including both local and systemic routes. Here we present the results available in the literature considering the mode of administration. This defines the 
level of the delivery challenge that needs to be achieved to obtain a therapeutic activity of the siRNA.

\subsection{Activity of siRNA delivered with chitosan-based nanoparticles by intratumoral administration}

The easiest way to achieve the delivery of a drug in a solid tumour is to perform intratumoral administration although this route of administration of drug is not relevant for clinical treatment. However, investigating new therapeutic strategies, intratumoral injection can serve the purpose of evaluating whether or not the new treatment can produce an anticancer activity in a straightforward manner.

De Martimprey et al. [56] have developed chitosan coated PIBCA nanospheres capable of carrying and protecting siRNA directed against RET/PTC1, a fusion oncogene involved in the tumorigenesis of radio induced thyroid cancer. In vitro, the shRNA transfected into cells with lipofectamine induced a high inhibition of the expression of the RET/PTC1 junction oncogene and even reversed the phenotype of murine NIH/3T3 cells bearing RET/PTC1 oncogene. Lipofectamine was used as transfection agent in the in vitro experiments because no inhibition of the target gene could be observed transfecting the cells with the siRNA-loaded chitosancoated PIBCA nanospheres. While the siRNA carried by the nanospheres was totally unable to silence the expression of the target gene in vitro for unknown reasons, the intratumoral delivery of the siRNA with these nanospheres produced a remarkable inhibition of the tumour growth [56]. In favour with the antitumoral activity, it was shown that the chitosan coated PIBCA nanospheres were efficient to protect the siRNA against nuclease degradation in the tumoral tissue over a period of 48 hours. In addition, the highlighted interfering activity that could be attributed to the siRNA indicated that the nanospheres were able to deliver the siRNA in the cancer cells.

Another anti-tumoral effect obtained from an interference activity targeting was reported after intra-tumoral injection of chitosan/anti-VEGF siRNA PEC nanoparticles in an experimental model of breast cancer in rats [57]. The reduction of the tumour volume which was $97 \%$ compared with non treated animals and control experiments were accompanied by a reduction of the VEGF protein as revealed by western blotting and immunohistochemistry. 


\subsection{Activity of siRNA delivered with chitosan-based nanoparticles after intravenous administration.}

Intravenous injections are so far, the most commonly used route for the administration of most anticancer treatments. This is because drugs can reach all kind of tissues including those inaccessible by other routes of administration. Intravenous injections are also considered to overcome absorption and degradation problems that can be encountered when drugs are administered by the oral route. Finally, the route of administration of drugs considering intravenous injections is also of clinical relevance when drugs need to reach disseminated tissues including metastasis for instance. Obviously, this route of administration needed to be considered for the delivery of anticancer treatment based on an interference strategy although designing such a treatment was extremely challenging. Indeed, it requires that the interfering agent, siRNA, will be efficiently protected against nuclease degradation by the carrier until it reaches the disease tissue and penetrate into cells to interact with the target mRNA. The carrier should have suitable properties to reach the diseased tissue after injection in the blood stream. For instance, it should be stable in the blood stream while the drug should remain associated with the carrier. It should also escape the immune system to have a chance to reach tissues containing the target cells otherwise it will be taken up by macrophages in charge of the elimination of foreign that are circulating in the blood $[58,60]$.

Finally, once in the diseased tissue, the interfering agent should reach the cytoplasm of the cells under an active form. Few studies aimed to evaluate the anti-tumoral activity of interfering agents delivered with chitosan-based PEC nanoparticles and chitosan-coated PIBCA nanospheres. They have clearly showed that the designed delivery systems were suitable to carry siRNA and shRNA in the blood stream and to achieve the delivery of the active molecules in the tumours [27,37,38]. This was acknowledged by the reduction of the tumour growth reported in the treated animals in comparison with untreated and control animals.

The chitosan coated PIBCA nanospheres carrying the siRNA against RET/PTC1 that showed anti-tumoral activity after intratumoral injection (see above) also showed an inhibition of the tumour growth following intravenous injection [37]. The inhibition of the tumour growth was correlated with an inhibition of the expression of the target gene $(\sim 40 \%)$ as shown by qPCR. Recently, Ramon et al. [39] reported similar results considering the delivery of a siRNA against EWS/Fli-1 with the same nanospheres and using an experimental model of Ewing sarcoma. Obviously, in both cases, the chitosan coated PIBCA nanospheres could deliver the 
active siRNAs in the tumour cells of the two experimental models. This meant that the siRNAs remained associated with the nanospheres while being transported in the blood stream from the site of administration to the tumour tissue and that the nanospheres could accumulate in the tumoral tissue thanks to the EPR effect. This also indicated that the nanoparticles carrying the siRNA were not taken up by the immune system. This in vivo fate agreed with the fact that the nanoparticles did not triggered the activation of the complement system which is part of the immune mechanism involved in the recognition of exogenous particles by macrophages [39]. According to additional experiments, the delivery of the siRNA in the tumoral tissue greatly depended on the dimension of the nanospheres which agreed with the principle of functioning of the EPR effect. The siRNA Ret/PTC1 delivered with the smaller chitosan-coated PIBCA nanospheres $(62 \mathrm{~nm})$ produced a higher inhibition of the tumour growth than when it was delivered with chitosan-coated PIHCA nanospheres which showed a larger diameter $(94 \mathrm{~nm})$. This difference in the capacity to induce tumour growth inhibition was attributed to a difference in the accessibility to tumoral tissue of the carried siRNA (Figure 11).

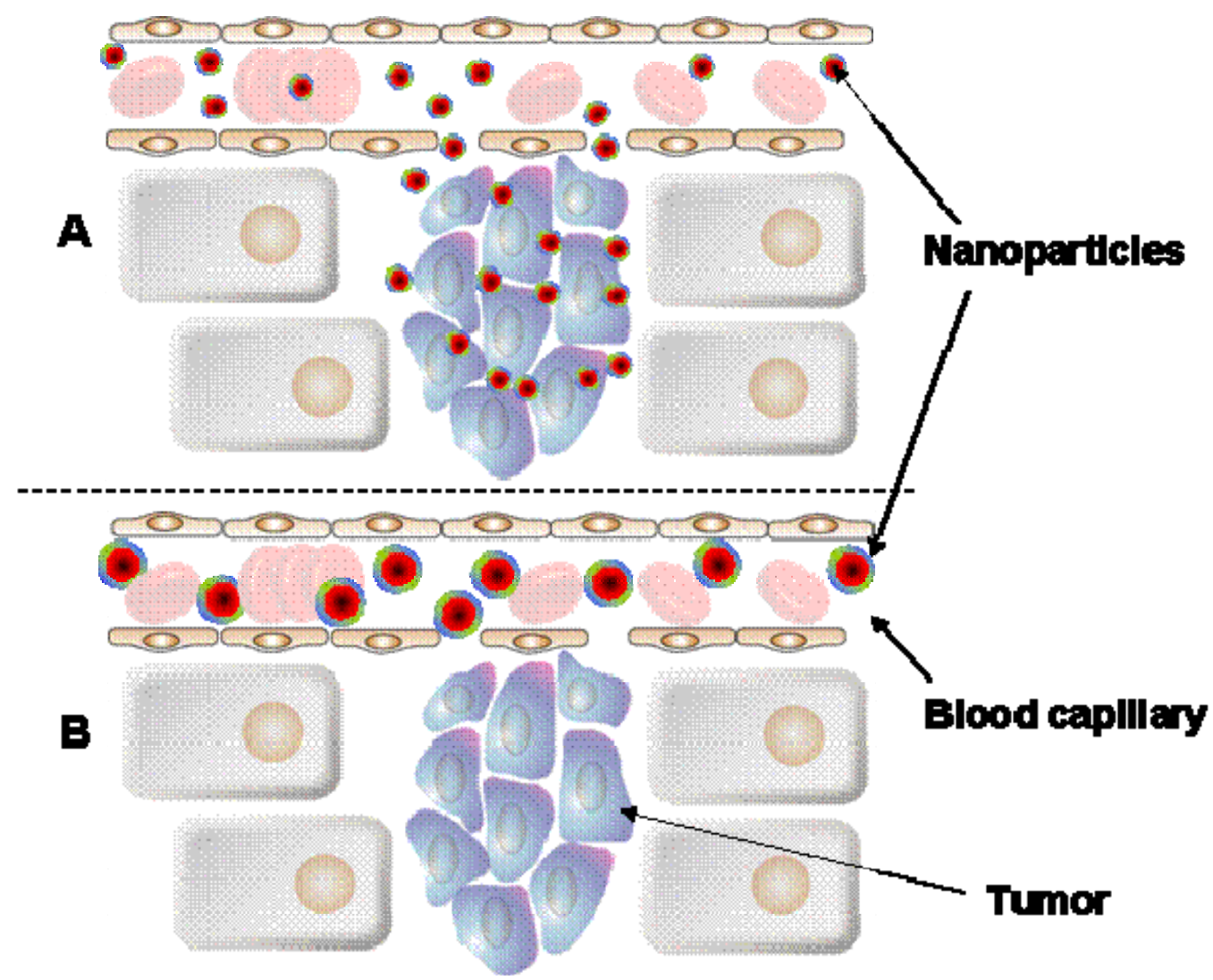

Figure 11: Scheme illustrating the uptake of the nanoparticles in the tumoral tissue from blood capillaries thanks to the enhancing permeability and retention effect. (A) siRNA-loaded PIBCA nanoparticles (diameter: $60.9 \pm 0.5 \mathrm{~nm}$ ), (B) siRNA-loaded PIHCA nanoparticles (diameter: $82 \pm 11$ ). Reproduced with permission [37]. 
The smaller nanospheres showed a diameter much below the pore size of fenestrations found in blood vessels feeding tumoral tissues. They could easily accumulate passively in the tumour thanks to the EPR effect. In contrast, the diameter of the larger nanospheres approached that of the fenestrations of vessels feeding the tumour tissue which could hamper the delivery of the siRNA into the tumoral tissue [61]. The smallest chitosan coated PIBCA nanospheres were used to design a targeted carrier for the siRNA active against the Ewing sarcoma. To this aim, antibodies targeting of a membrane protein over-expressed in Ewing's sarcoma cells from human origin were grafted on the chitosan corona of the nanospheres. As a result, from the in vivo evaluation, the siRNA injected by the intravenous route associated with the targeted nanospheres was more efficient to inhibit the fusion oncogene EWS/Fli-1 in comparison with the delivery achieved with the non-targeted nanospheres [39]. Results from a preliminary study of the in vivo biodistribution of the siRNA targeted nanospheres showed that the siRNA delivered with the targeted nanospheres accumulated to a greater extend in the tumour compared to their delivery with the untargeted nanospheres [62]. All together, these results confirmed the potential of chitosan coated PIBCA nanospheres as carriers and transfection agent for siRNA in vivo after intravenous injection. Another carrier evaluated for the delivery of siRNA by the intravenous route included targeted nanogels [28].

The nanogels used in the study of Han et al. [28] were designed using chitosan grafted with the tripeptide Arg-Gly-Asp (RGD) and TPP as the crosslinker. The RGD peptide is a wellknown ligand used to target the $\alpha v \beta 3$ integrin over-expressed on the membrane of various types of cancer cells and tumour vasculature [63]. This integrin was identified as an interesting cell surface receptor to target in drug delivery strategy because it can facilitate the internalization of the drug [64]. The designed targeted nanogels were used to deliver a siRNA directed against the gene periostin (POSTN). This gene is over-expressed in the ovarian cancer cell line SKOV3ip1 that was used to develop an orthotopic in vivo model of ovarian cancers in mice. These cells that also over-expressed the $\alpha v \beta 3$ integrin were relevant to explore the targeting strategy with the RGD peptide grafted on the siRNA loaded nanogel. This was verified in vitro by showing that the RGD targeted nanogels enhanced the binding efficiency to the SKOV3ip1. In vivo, 24 hours after a single intravenous injection of the siRNA associated with the targeted nanogel, the reduction of the expression of the gene POSTN was above 51\% compared with a treatment performed with the non-loaded nontargeted nanogel which has no effect on tumour growth and compared to untreated animals. 
This inhibition of the expression of the target gene of the siRNA was much higher than the inhibition measured on animals treated with the siRNA delivered with the non targeted nanogel. In this case, the inhibition of the gene POSTN was only $20 \%$ compared with a treatment performed with the non-loaded non-targeted nanogel. In parallel to the reduction of the expression of the gene POSTN produced by the siRNA delivered with the targeted nanogel, a significant reduction of the tumour growth was reported. These results could be correlated with the effective delivery of the siRNA in the tumour cells as shown by a colocalization of the fluorescent-labelled siRNA and the $\alpha v \beta 3$ integrin in the tumoral tissue revealed after intravenous administration of the fluorescently labelled Alexa555 siRNA loaded RGD targeted nanogel. Interestingly, the addition of an anticancer agent such as docetaxel in the siRNA loaded RGD nanogels enhanced the antitumoral effect of the designed targeted interference treatment.

\subsection{Activity of siRNA delivered with chitosan-based nanoparticles considering an administration via the respiratory tract.}

Administration of drugs by the respiratory tract allows delivery of actives to both the respiratory system and the blood. This route of administration is non invasive and allows to bypass the first hepatic passage discarding the risk of clearance of drugs by the liver when used as a systemic route of drug delivery. It was considered for the delivery of siRNA and shRNA in several studies mainly for local action.

A first study was aimed to evaluate the delivery of a siRNA designed to inhibit the expression of the green fluorescent protein gene. The treatment consisted in daily intranasal administration of chitosan/siRNA PEC nanoparticles for 5 days [65]. While the treatment was well tolerated and no general toxicity was highlighted, an inhibition of the expression of the green fluorescent proteins was monitored in epithelial cells of the lung bronchioles. The percentage of the inhibition was $43 \%$ compared with the expression measured in untreated animals. By aerosolization of chitosan/Cy3-labeled siRNA PEC nanoparticles with an intratracheal catheter, Gianfelici et al. showed that the siRNA delivered with the PEC nanoparticles could reached the entire lung and inhibit the expression of the fluorescent green protein by $68 \%$ compared with control experiments [66]. Results from these works have demonstrated that siRNA can be delivered locally to the lung under an active form with PEC nanoparticles. They are encouraging to pursue the research considering relevant models of lung diseases. 
As already mentioned, the pulmonary route can by used to deliver drugs to the systemic circulation. However, it has not been considered for the systemic delivery of interfering molecules so far.

\subsection{Activity of siRNA delivered with chitosan-based nanoparticles after oral delivery}

The oral route is non-invasive and can be used to deliver active molecules to the systemic circulation. It has many advantages being the most accepted by patients, safe and cost effective. However, it is the most challenging for many drugs. Many molecules are indeed degraded in the harsh conditions found in the gastrointestinal tract. Even if the molecule resists to degradation their absorption can be compromised due to the highly selective absorptive barrier formed by the gut epithelium. Together, these conditions hampered the absorption of many drugs in their active form. The chemical structure and physicochemical properties shown by the interfering compounds including siRNA are not favourable for their administration by the oral route especially when it is expected that they should reach the systemic circulation. Nevertheless, few authors have very recently tested the delivery of siRNA by the oral route considering their formulation in chitosan-based nanoparticle as delivery systems.

One work was aimed to develop a targeted interference treatment of ulcerative colitis which is a chronic inflammatory bowel disease [25]. The strategy followed by the authors was to target macrophages thanks to the attachment of a galactose residue on the siRNA carrier and to develop the interference activity by knocking down the expression of the mitogen-activated protein kinase 4 (Map4k4) gene, which was shown to induce a suppression of the production of TNF- $\alpha$. The siRNA delivery system designed for this purpose was a nanogel formed by the association of the siRNA with galactosylated modified chitosan and TPP. The modified chitosan used was trimethylchitosan including cysteines residues. With their thiol groups, the cysteine residues were added to potentialize the mucoadhesiveness of the chitosan based nanogels [67]. The siRNA associated with the nanogels formed particles with a rather small size (150 $\mathrm{nm}$ in diameter) while the siRNA resisted well against degradation for an incubation of 6 hours in serum and in reconstituted intestinal fluids. After oral gavage to an experimental model of colitis developed in mice, the Map4K4 siRNA was able to suppress the expression of Map4k4 and the production of TNF- $\alpha$ in the colonic tissue by $92.1 \%$ and $69 \%$ respectively only when it was administered with the targeted and non-targeted nanogels. No suppression of 
the production of TNF- $\alpha$ was monitored in mice treated with the scramble siRNA associated with the same nanogels and used as a control. Interestingly, the histological examination of the tissues recovered from the mice treated with the Map4K4 siRNA associated with the nanogels revealed significant improvements compared with those recovered from groups of animals of the control experiments. All together the results of this study were very encouraging to continue the development of this potential orally administrated interfering treatment of ulcerative colitis. Other arguments in favor of pursuing this work is the ease of the preparation of the formulation and the much lower cytotoxicity found for the siRNAloaded nanogels compared with previously formulated systems [28].

A series of very recent works proposed by different authors reported results obtained from experiments designed to deliver siRNA at the systemic level from an oral administration [33, 68, 69]. The three works were carried out considering different chitosan-based delivery systems that were administered by oral gavage to animals. These included chitosan/siRNA PEC nanoparticles [69], nanogels [68] and oil containing nanocapsules [32]. In the three cases, the chitosan-based delivery system protected well the siRNA against degradation in serum and in gastro-intestinal fluids. Importantly, all these systems could deliver intact siRNA in tissues that can only be reached by the systemic circulation after absorption by the intestinal tissue. Ballarin-Gonzalez et al. [68] who have worked with the simpler system have pointed out the importance of the N/P ratio used to form the chitosan/siRNA PEC nanoparticles to achieve a sufficient stability of the assembly making possible the delivery of the intact siRNA to the liver, spleen and kidneys. The siRNA reached these tissues, only accessible by systemic translocation, 1 hour after oral gavage. Although results from this work support the validity of the delivery method, it was not in the aim of this study to demonstrate an interfering activity at this stage. In the two other studies, siRNA with relevant interfering activities were tested in oral administration conditions for their systemic activity on experimental models of diseases. A TNF- $\alpha$ siRNA was delivered with nanogels [69]. The nanogels was designed with TPP as the reticulating agent and a modified chitosan which included mannose residues for the targeting of macrophages, trimethyl group to reinforce the positive charges of chitosan for a better complexation with siRNA and cysteine residue to improve the mucoadhesion and confer enhancing absorption properties in the gastrointestinal tract. This system was tested on an experimental model of acute hepatic injury induced by LPS/D-GalN in mice. The results indicated that the active siRNA could reach different organs including the liver, the spleen and the lungs and infiltrate reticuloendothelial tissues. Thus, the 
siRNA delivered by the nanogels could permeate across the intestinal epithelium and traffic through the systematically circulation until they reached the tissues. Moreover, these nanogels loaded with the TNF- $\alpha$ siRNA mediated gene silencing activity and blocked the appearance of the hepatic damaged expected to appear after induction by the administration of the LPS/DGalN in the model of acute hepatic injury. From other results, the siRNA nanogels used in this study appeared safe as acknowledged by the lack of innate immune response and a good tolerance regarding the haematological parameters and the evaluation of several biochemical parameters. These nanogels are exiting systems for the development of a treatment of systemic inflammatory diseases with a therapeutic method based on interfering approach with siRNA delivered by the oral route.

Success in the systemic delivery of siRNA by the oral route is also very attractive for the development of anticancer treatments. Such a strategy was explored by Wei et al. [33] using a siRNA directed against the mouse telomerase reverse transcriptase (mTERT) and administered with chitosan based nanocapsules. The nanocapsules were fabricated from oilin-water-in-oil (o/w/o) emulsion by reticulating the modified chitosan $\mathrm{N}-((2$-hydroxy-3trimethylammonium) propyl) chitosan chloride (HTCC) with glutaraldehyde prior to the association of the siRNA. To give the nanocapsules suitable surface properties and to promote mucoadhesion on the gut mucosa and absorption, a supplemental layer of HTCC was adsorbed on the surface of the siRNA-loaded nanocapsules. Finally, the oily core of the nanocapsules was loaded with paclitaxel which is an anticancer agent applied in clinics in many chemotherapy protocols used to treat various cancers. The final nanocapsules displayed a size of 130 to $145 \mathrm{~nm}$, which remained in a small range. As reported with the previous systems, these nanocapsules were also able to deliver the active siRNA to the systemic circulation. More interestingly, the antitumoral activity monitored by the measurement of the tumour growth and by the evaluation of the survival of the animals was attributed to the interference activity of the siRNA having reached the tumoral tissue. The evaluation of the antitumoral activity was achieved in an experimental model of Lewis lung carcinoma implanted in mice by subcutaneous injection while the treatments were given by oral gavage. It is noteworthy that the antitumoral activity was undisputable. Control treatments performed by oral gavage of the naked siRNA had no effect on the tumour growth compared with a treatment performed with a saline solution (the increase of the relative tumour volume was 18 $\%$ considering the siRNA and 20\% with the saline solution after 11 and 12 days respectively) and after 11 to 12 days all mice treated with siRNA and the saltine solution respectively had 
died. In contrast, treatment with the nanocapsules conjugating the action of the siRNA directed against mTERT and paclitaxel in the same carrier led to almost $90 \%$ survival after 15 days and the increase of the tumour volume was only $5 \%$ over the same period of time. The delivery of paclitaxel and of the siRNA in separate nanocapsules showed a higher antitumoral activity than the control experiments but the antitumoral activity remained lower than that of the two-in-one nanocapsule formulation. The antitumoral activity of the nanocapsules was well correlated with a silencing effect of the mTERT gene at both the level of the mRNA and the level of the protein as demonstrated by Q-PCR and western blot analysis performed on the tumoral tissue. The knock down efficiency was estimated to have occurred as up to 3 folds in RT-PCR with the treatment performed with the nanocapsules combining the siRNA and PTX compared with treatments performed with nanocapsules loaded either with siRNA alone or with paclitaxel alone and with a treatment in which both types of nanocapsules were mixed. The anticancer activity was supported by a favorable in vivo fate of the nanocapsules after oral administration. The nanocapsules were able to deliver the siRNA and paclitaxel to the tumour as acknowledged by the results of the various experiments designed to investigate the absorption of the drug molecules and the system by the intestinal tract and the biodistribution of the siRNA and paclitaxel after oral gavage in the mice bearing tumour. It was also clearly showed that the two-in-one nanocapsules were more efficient to achieve a joint delivery of the two molecules then a blend of nanocapsules that were each loaded with one type of drug. This first proof-of-concept demonstrated that an efficient anticancer treatment based on the oral delivery of siRNA can be achieved. The nanocapsules and especially their method of preparation may present some difficulties for a development in clinics. Nevertheless, this work opens exiting perspectives for a new generation of cancer treatment.

Collectively, these three examples have demonstrated the feasibility of the administration of interfering therapy by the oral route thanks to the use chitosan based nanosized delivery systems even for a systemic delivery. It is noteworthy that the obtaining an antisens effect from a delivery of siRNA by the oral route was not straightforward. Indeed, the physicochemical properties of siRNA are unfavourable to expect a good absorption through the gastrointestinal mucosa. The observation of an antitumoral effect and of therapeutic activities related to the interfering effect of the siRNA at a systemic level suggested that a sufficient amount of siRNA was absorbed and reached the target cells. Although the mechanism of the oral delivery of active siRNA needs to be elucidated, several assumptions can be drawn to explain this success. Chitosan displays mucoadhesive and absorption enhancing properties. 
Both are exploited to design mucoadhesive formulations of molecules like peptides, proteins and DNA for the oral route including under the form of nanoparticles [70,71]. Based on these works, it can be assumed that the nanoparticles formulated with chitosan could facilitate the absorption of siRNA. The fact that the siRNA was active after systemic delivery following oral administration indicated that the siRNA was protected from the degradation by nucleases while it was transported by the blood towards the siRNA target cells. This suggested that the siRNA remained associated with chitosan and perhaps with the nanoparticles while absorbed by the gut and transported through the blood stream down to their target cells. Specific works are needed to explain this unexpected effect which opens exiting perspectives for the development of treatments by the oral route which is the safer and most accepted for patients.

\section{Conclusion}

This review focuses on chitosan-based nanoparticle carriers for in vivo delivery of interfering molecules including siRNA. As pointed out, a few types of chitosan-based nanoparticles that differ from their structures were developed as carriers for the delivery of interfering agents. In these systems, chitosan or its derivatives play central roles controlling the association of siRNA and the stability of the nanoparticles, the release of the siRNA and the stability of the siRNA against enzymatic degradation. Properties of chitosan and conditions of associations with siRNA influence the potential of the system to deliver siRNA producing an interference activity in vitro and in vivo. Further work remains to elucidate the role of each parameter influencing the delivery of the biologically active. Besides, the potential of the delivery systems was clearly improved by adding a targeting ligand on the surface of the chitosanbased nanoparticles in charge of the delivery of the interfering molecule. This strategy increases the efficacy of delivering siRNA to the target cells and is expected to promote the required intracellular delivery of the siRNA. Very recent studies have demonstrated that siRNA-loaded chitosan-based nanoparticles are suitable to induce an interference activity at the systemic level from an oral administration. Attempt to deliver siRNA by the oral route was an extremely high challenge and the reported successes undoubtedly paved the road for promising developments of treatments based on siRNA in the future by this route of administration. This gives very exiting perspectives. However, several challenges still need to be addressed. For instance, issues regarding the safety profile of the chitosan-based nanoparticle carriers used for siRNA delivery have not yet been fully clarified and further works need to be performed to clear out this remaining question. Another issue making difficult the development of these systems is due to the fact that successes reported from in 
vivo tests could not always be anticipated from in vitro tests that led to inconsistent and discouraging results. This situation also mentioned in the case of gene delivery with chitosanbased nanoparticles, appeals for giving priority to tests of the efficacy of the siRNA delivery method based on the use of in vivo models. This obviously complicates the identification of new potential treatments based on the delivery of siRNA formulated with chitosan-based nanoparticles especially because there are many factors coming influencing the delivery method that will need to be investigated. In addition, requirements associated with such a strategy are pending on the availability of corresponding animal models. On a more general point of view, the design of highly specific and effective siRNA at a low dose can be identified as a bottleneck that hamper rapid development of treatments based on siRNA. This has been identified as the issue to contour toxicity of siRNA related to off target undesired effects. SiRNAs being active at very a low dose and targeting the region of mRNAs including the mutation point or the junction region of a junction oncogene may be suitable solutions to overcome this obstacle. Despite these difficulties, today chitosan-based nanoparticles show the best compromise in term of safety and in vivo delivery efficacy of siRNA. Efforts will be pursued for their developments to propose innovative treatments based

\section{References}

[1] Sen GL, Blau HM. A brief history of RNAi: the silence of the genes. FASEB J 2006;20: 1293-9. * History about the discovery of the RNA interference silencing gene effect

[2] Huang PI, Lo WL, Cherng JY, Chien Y, Chiou GY, Chiou SH. Non-viral delivery of RNA interference targeting cancer cells in cancer gene therapy. Curr Gene Ther 2012; 12:27584 . $* *$ Report on artificial delivery systems for RNA interference therapeutics of cancer including an update on ongoing clinical developments

[3] Rao DD, Vorhies JS, Senzer N, Nemunaitis J. siRNA vs. shRNA: similarities and differences. Adv Drug Deliv Rev 2009;61:746-59.

[4] Khatri N, Rathi M, Baradia D, Trehan S, Misra A. In vivo delivery aspects of miRNA, shRNA and siRNA. Crit Rev Ther Drug Carrier Syst 2012;29:487-527.**Review on the delivery of various types of interfering molecules pointed out challenges and potential methods developed to achieve in vivo delivery

[5] Zhang S, Zhi D, Huang L. Lipid-based vectors for siRNA delivery. J Drug Target. 2012;20:724-35. * Review paper on lipid based delivery systems for siRNA

[6] Boussif O, Lezoualc'h F, Zanta MA., Mergny MD, Scherman D, Demeneix B et al. A versatile vector for gene and oligonucleotide transfer into cells in culture and in vivo: polyethylenimine. Proc Natl Acad Sci USA 1995;92:7297-301.*Pionnier work on the development of artifitial carriers for delivery of nucleic acids based on the formation of polyelectrolyte complexes.

[7] Zaric V, Weltin D, Erbacher P, Remy JS, Behr JP, Stephan D. Effective polyethyleniminemediated gene transfer into human endothelial cells. J Gene Med 2004;6:176-84. 
[8] Bruno K. Using drug-excipient interactions for siRNA delivery. Adv Drug Deliv Rev 2011;63:1210-26.

[9] Howard KA. Delivery of RNA interference therapeutics using polycation-based nanoparticles. Adv Drug Deliv Rev 2009;61:710-20.**Review articles summarizing advances in the development of delivery systems for RNA interference therapeutics based on the use of nanoparticles designed with polycations

[10] Raviña M, Cubillo E, Olmeda D, Novoa-Carballal R, Fernandez-Megia E, Riguera R, et al. Hyaluronic acid/chitosan-g-poly(ethylene glycol) nanoparticles for gene therapy: an application for pDNA and siRNA delivery. Pharm Res 2010;27:2544-55.

[11] Yuan X, Naguib S, Wu Z. Recent advances of siRNA delivery by nanoparticles. Expert Opin Drug Deliv 2011;8:521-36.

[12] Duceppe N, Tabrizian M. Advances in using chitosan-based nanoparticles for in vitro and in vivo drug and gene delivery. Expert Opin Drug Deliv. 2010;7:1191-207.

[13] Raftery R, O'Brien FJ, Cryan SA, Chitosan for Gene Delivery and Orthopedic Tissue Engineering Applications. Molecules 2013;18:5611-47.

[14] Al-Qadi S, Grenha A, Remunan-Lopez C. Chitosan and its derivatives as nanocarriers for siRNA delivery. J Drug Del Sci Technol 2012;22:29-42.

[15] Mao S, Sun W, Kissel T. Chitosan-based formulations for delivery of DNA and siRNA. Adv Drug Deliv Rev 2010;62:1227.

[16] Rudzinski WE, Aminabhavi TM. Chitosan as a carrier for targeted delivery of small interfering RNA. Int J Pharm 2010;399:1-11.

[17] Rodrigues S, Dioniso M, Remunan-Lopez C, Grenha A. Biocompatibility of chitosan carriers with applications in drug delivery. J Funct Biomater 2012;3:615-41.

[18] Kim YK, Jiang HL, Guo DD, Choi YJ, Cho MH, Akaike T, Cho CS. Chemical Modification of Chitosan for Delivery of DNA and siRNA. in: Sarmento B, Das Neves J, editors. Chitosan-Based Systems for Biopharmaceuticals, New York: John Wiley \& Sons, Ltd, 2012, p. 255-73. **Reviews of all the modifications applied on chitosan modulating properties to improve gene delivery.

[19] Drogoz A, David L, Rochas C, Domard A, Delair T. Polyelectrolyte complexes from polysaccharides: formation and stoichiometry monitoring. Langmuir 2007;23:109508.**outstanding work on the formation of PEC nanoparticles

[20] Katas H, Alpar HO. Development and characterization of chitosan nanoparticles for siRNA delivery. J Control Release 2006;115:216-25.

[21] Dehousse V, Garbacki N, Jaspart S, Castagne D, Piel G, Colige A et al. Comparison of chitosan/siRNA and trimethylchitosan/siRNA complexes behaviour in vitro. Int J Biol Macromol 2010; 46: 342-9.

[22] Techaarpornkul S, Wongkupasert S, Opanasopit P, Apirakaramwong A, Nunthanid J, Ruktanonchai U. Chitosan-mediated siRNA delivery in vitro: effect of polymer molecular weight, concentration and salt forms. AAPS Pharm Sci Tech 2010;11:64-72.

[23] Dehousse V, Garbacki N, Colige A, Evrard B. Development of pH-responsive nanocarriers using trimethylchitosans and methacrylic acid copolymer for siRNA delivery. Biomaterials 2010;31:1839-49.

[24] Park S, Jeong EJ, Lee J, Rhim T, Lee SK, Lee KY. Preparation and characterization of nona-arginine-modified chitosan nanoparticles for siRNA delivery. Carbohydr Polym 2013;92:57-62. 
[25] Zhang J, Tang C, Yin C. Galactosylated trimethyl chitosan-cysteine nanoparticles loaded with Map4k4 siRNA for targeting activated macrophages. Biomaterials 2013;34:366777.**Multifunctional system for systemic delivery of siRNA by the oral route. Remarkable systemic interfering activity of the siRNA was reported after delivery of the siRNA formulated in nanogels by the oral route.

[26] He P, Davis SS, Illum L. In vitro evaluation of the mucoadhesive properties of chitosan microspheres. Int J Pharm 1998;166:75-88.

[27] Malhotra M, Tomaro-Duchesneau C, Prakash S. Synthesis of TAT peptide-tagged PEGylated chitosan nanoparticles for siRNA delivery targeting neurodegenerative diseases. Biomaterials 2013;34:1270-80.

[28] Han HD, Mangala LS, Lee JW, Shahzad MMK, Kim HS, Shen D et al. Targeted gene silencing using RGD-labeled chitosan nanoparticles. Clin Cancer Res 2010;16:3910-22.

[29] Abbas AO, Donovan MD, Salem AK. Formulating poly(lactide-co-glycolide) particles for plasmid DNA delivery. J Pharm Sci 2008;97:2448-61.25 Nafee N, Taetz S, Schneider M, Schaefer UF, Lehr CM. Chitosan-coated PLGA nanoparticles for DNA/RNA delivery: effect of the formulation parameters on complexation and transfection of antisense oligonucleotides. Nanomedicine 2007;3:173-83.

[30] Nafee N, Taetz S, Schneider M, Schaefer UF, Lehr CM. Chitosan-coated PLGA nanoparticles for DNA/RNA delivery: effect of the formulation parameters on complexation and transfection of antisense oligonucleotides. Nanomedicine 2007;3:17383.

[31] Tahara K, Yamamoto H, Hirashima N, Kawashima Y. Chitosan-modified poly(D,Llactide-co-glycolide) nanospheres for improving siRNA delivery and gene-silencing effects. Eur J Pharm Biopharm 2010;74:421-6.

[32] Toub N, Bertrand JR, Tamaddon A, Elhamess H, Hillaireau H, Maksimenko A et al. Efficacy of siRNA Nanocapsules Targeted Against the EWS-Flil Oncogene in Ewing Sarcoma. Pharm Res 2006;23:892-900.

[33] Wei W, Lv PP, Chen XM, Yue ZG, Fu Q, Liu SY et al. Codelivery of mTERT siRNA and paclitaxel by chitosan-based nanoparticles promoted synergistic tumour suppression. Biomaterials 2013;34:3912-23. **Work considering the development of a coencapsulation of an anticancer agent and an interfering agent with anticancer activity for a co-administration by the oral route. Demonstration of the obtaining of a systemic effect of both the anticancer agent and the interfering molecule.

[34] Maksimenko A, Polard V, Villemeur M, Elhamess H, Couvreur P, Bertrand JR et al. In vivo potentialities of EWS-Fli-1 targeted antisense oligonucleotides-nanospheres complexes. Ann. N. Y. Acad. Sci. 2005;1058:52-61.

[35] Bertholon I, Vauthier C, Labarre D. Complement activation by core-shell poly(isobutylcyanoacrylate)-polysaccharide nanoparticles: influences of surface morphology, length, and type of polysaccharide. Pharm Res 2006 ;23:1313-23.

[36] Bravo-Osuna I, Ponchel G, Vauthier C. Tuning of shell and core characteristics of chitosan-decorated acrylic nanoparticles. Eur J Pharm Sci 2007;30:143-54.

[37] De Martimprey H, Bertrand JR, Malvy C, Couvreur P, Vauthier C. New core-shell nanoparticules for the intravenous delivery of siRNA to experimental thyroid papillary carcinoma. Pharm Res 2010,27:498-509. 
[38] Zandanel C, Vauthier C. Poly(isobutylcyanoacrylate) Nanoparticles Decorated with Chitosan: Effect of Conformation of Chitosan Chains at the Surface on Complement Activation Properties. J Coll Sci Biotechnol 2012;1:68-81.

[39] Ramon AL, Bertrand JR, De Martimprey H, Bernard G, Ponchel G, Malvy C et al. SiRNA associated with immunonanoparticles directed against cd99 antigen improve gene expression inhibition in vivo in Ewing's sarcoma. J Mol Recognit 2013;26:318-29.

[40] Zandanel C, Vauthier C. Characterization of fluorescent poly(isobutylcyanoacrylate) nanoparticles obtained by copolymerization of a fluorescent probe during Redox Radical Emulsion Polymerization (RREP). Eur J Pharm Biopharm 2012;82:66-75.

[41] Holzerny P, Ajdini B, Heusermann W, Bruno K, Schuleit M, Meinel L et al. Biophysical properties of chitosan/siRNA polyplexes: Profiling the polymer/siRNA interactions and bioactivity. J Control Release 2012; 157:297-304

[42] Zandanel C, Noiray M, Vauthier C. Chitosan-decorated poly(isobutylcyanoacrylate nanoparticles: a platform for siRNA delivery. Proceedings of the 16th International Symposium on Recent Advances in Drug Delivery Systems. Salt Lake City, UT, USA, 36 February 2013. p. 89-90.

[43] Xu S, Dong M, Liu X, Howard KA, Kjems J, Besenbacher F. Direct force measurements between siRNA and chitosan molecules using force spectroscopy. Biophys $\mathrm{J}$ 2007;93:952-9.

[44] López-León T, Carvalho ELS, Seijo B, Ortega-Vinuesa JL, Bastos-González D. Physicochemical characterization of chitosan nanoparticles: electrokinetic and stability behavior. J Colloid Interface Sci 2005;283:344-51.

[45] Liu X, Howard KA, Dong M, Andersen MØ, Rahbek UL, Johnsen MG et al. The influence of polymeric properties on chitosan/siRNA nanoparticle formulation and gene silencing. Biomaterials 2007;28:1280-8.

[46] Lee DW, Yun KS, Ban HS, Choe W, Lee SK, Lee KY. Preparation and characterization of chitosan/polyguluronate nanoparticles for siRNA delivery. J Control Release 2009;139:146-52.

[47] Fernandes JC, Qiu X, Winnik FM, Benderdour M, Zhang X, Dai K et al. Low molecular weight chitosan conjugated with folate for siRNA delivery in vitro: optimization studies. Int J Nanomedicine 2012;7:5833-45.

[48] Taetz S, Nafee N, Beisner J, Piotrowska K, Baldes C, Mürdter TE et al. The influence of chitosan content in cationic chitosan/PLGA nanoparticles on the delivery efficiency of antisense 2'-O-methyl-RNA directed against telomerase in lung cancer cells. Eur J Pharm Biopharm 2009;72:358-69.

[49] Yuan X, Shah BA, Kotadia NK, Li J, Gu H, Wu Z. The development and mechanism studies of cationic chitosan-modified biodegradable PLGA nanoparticles for efficient siRNA drug delivery. Pharm Res 2010;27:1285-95.

[50] Howard KA. Delivery of RNA interference therapeutics using polycation-based nanoparticles. Adv Drug Deliv Rev 2009;61:710-20.

[51] Thibault M, Nimesh S, Lavertu M, Buschmann MD. Intracellular trafficking and decondensation kinetics of chitosan-pDNA polyplexes. Mol Ther 2010;18:1787-95.

[52] He C, Yin L, Tang C, Yin C. Multifunctional polymeric nanoparticles for oral delivery of TNF-alpha siRNA to macrophages. Biomaterials 2013;34:2843-54. 
[53] Malmo J, Sandvig A, Varum KM, Strand SP. Nanoparticle mediated P-glycoprotein silencing for improved drug delivery across the blood-brain barrier: a siRNA-chitosan approach. PLoS One 2013;8:e54182.** very interesting approach for promoting drug delivery to the brain

[54] Tu Q, Zhang Y, Liu R, Wang JC, Li L, Nie N et al. Active drug targeting of disease by nanoparticles functionalized with ligand to folate receptor. Curr Med Chem 2012;19:3152-62.

[55] Lee J, Yun KS, Choi CS, Shin SH, Ban HS, Rhim T et al. T Cell-Specific siRNA Delivery Using Antibody-Conjugated Chitosan Nanoparticles. Bioconjug Chem 2012;23:1174-80.

[56] De Martimprey H., Bertrand JR, Fusco A, Santoro M, Couvreur P, Vauthier C et al. siRNA nanoformulation against the ret/PTC1 junction oncogene is efficient in an in vivo model of papillary thyroid carcinoma. Nucleic Acids Res 2008;36: e2.

[57] Salva E, Kabasakal L, Eren F, Ozkan N, Cakalagaoglu F, Akbuga J. Local delivery of chitosan/VEGF siRNA nanoplexes reduces angiogenesis and growth of breast cancer in vivo. Nucleic Acid Ther 2012;22:40-8.

[58] Owens DE, Peppas NA. Opsonization, biodistribution, and pharmacokinetics of polymeric nanoparticles.Int J Pharm. 2006;307:93-102.

[59] Vonarbourg A, Passirani C, Saulnier P, Benoit JP. Parameters influencing the stealthiness of colloidal drug delivery systems. Biomaterials. 2006;27:4356-73.

[60] Amoozgar Z, Yeo Y. Recent advances in stealth coating of nanoparticle drug delivery systems. Wiley Interdiscip Rev Nanomed Nanobiotechnol. 2012;4:219-33.

[61] Decuzzi P, Pasqualini R, Arap W, Ferrari M. Intravascular delivery of particulate systems: does geometry really matter? Pharm Res 2009;26:235-43.

[62] Ramon AL, Bertrand JR, Zandanel C, Vauthier C, Malvy C. Biodistribution of siRNA delivered with chitosan-decorated poly(isobutylcyanoacrylate) nanoparticles. Proceedings of the 16th International Symposium on Recent Advances in Drug Delivery Systems. Salt Lake City, UT, USA, 3-6 February 2013. p. 66-67.

[63] Garanger E, Boturyn D, Dumy P. Tumour targeting with RGD peptide ligands-design of new molecular conjugates for imaging and therapy of cancers. Anticancer Agents Med Chem 2007;7:552-8.

[64] Hood JD, Bednarski M, Frausto R, Guccione S, Reisfeld RA, Xiang R et al. Tumour regression by targeted gene delivery to the neovasculature. Science 2002;296:2404-7.

[65] Howard KA, Rahbek UL, Liu X, Damgaard CK, Glud SZ, Andersen MO et al. RNA interference in vitro and in vivo using a novel chitosan/siRNA nanoparticle system. Mol Ther 2006;14:476-84.

[66] Gianfelici V, Lahortiga I, Cools J. Chromosomal aberrations and fusion genes in myeloid malignancies. Expert Rev Hematol 2012; 5:381-93.

[67] Hauptstein S, Bernkop-Schnürch A. Thiomers and thiomer-based nanoparticles in protein and DNA drug delivery. Expert Opin Drug Deliv. 2012;9:1069-81.

[68] Ballarín-González B, Dagnaes-Hansen F, Fenton RA, Gao S, Hein S, Dong M et al. Protection and systemic translocation of siRNA following oral administration of chitosan/siRNA nanoparticles. Mol Ther Nucleic Acids 2013 Mar 5;2:e76. doi: 10.1038/mtna.2013.2. 
[69] He C, Yin L, Tang C, Yin C. Multifunctional polymeric nanoparticles for oral delivery of TNF- $\alpha$ siRNA to macrophages. Biomaterials 2013;34:2843-54.* report on systemic activity of siRNA delivered with multifunctional polymeric nanoparticles by the oral route.

[70] Bowman K, Leong KW. Chitosan nanoparticles for oral drug and gene delivery. Int J Nanomedicine. 2006;1:117-28.

[71] Chaudhury A, Das S. Recent advancement of chitosan-based nanoparticles for oral controlled delivery of insulin and other therapeutic agents. AAPS PharmSciTech. 2011;12:10-20. 